\title{
Thioxopyrimidine in Heterocyclic Synthesis II: Novel Synthesis of Some Triazoles and Triazepine Derivatives with a Pyrimido[3,2:4,5] thieno[2,3-d]pyrimidine Skeleton
}

\author{
Yuh Wen Ho and Se Long Chou \\ Department of Creative Fashion Design, Taoyuan Institute of Innovation Technology, Jhongli 32091, Taiwan \\ Correspondence should be addressed to Yuh Wen Ho; wen@tiit.edu.tw
}

Received 28 June 2012; Accepted 19 September 2012

Academic Editor: Diego Sampedro

Copyright (C) 2013 Y. Wen Ho and S. L. Chou. This is an open access article distributed under the Creative Commons Attribution License, which permits unrestricted use, distribution, and reproduction in any medium, provided the original work is properly cited.

Condensation of ethoxymethyleneamino-thieno[2,3- $d$ ]pyrimidines 4 with appropriate amino compounds afforded the corresponding 7-substituted-8-imino-pyrimido[3,2:4,5]thieno[2,3- $d]$ pyrimidines $\mathbf{6 a}, \mathbf{6 b}$, and 7 and 2-substitutedpyrimido $\left[3^{\prime}, 2^{\prime}: 4,5\right]$ thieno[3,2-e][1,2,4] triazolo[1,5-c]pyrimidines $\mathbf{9 a}, \mathbf{9 b}$, respectively. Also, hydrazinolysis of compound 4 in ethanol which yielded the key intermediate 7-amino-8-imino-pyrimido[3,2:4,5]thieno[2,3-d]pyrimidine (10), which can be cyclized with appropriate isothiocyanates 14a-14g in refluxing pyridine, afforded the corresponding 2-(substitutedamino)-pyrimido $\left[3^{\prime}, 2^{\prime}: 4,5\right]$ thieno[3,2-e][1,2,4] triazolo[1,5-c]pyramidines 15a-15g. Furthermore, intramolecular cyclization of compound (10) with appropriate 1,3-dibromopropane and Mannich bases 18a-18c under the basic condition afforded the corresponding (tri)dihydropyrimido $\left[3^{\prime}, 2^{\prime}: 4,5\right]$ thieno[3,2:4,5]pyrimido[1,6-b][1,2,4]triazepines $\mathbf{1 6}$ and $\mathbf{1 8 a}-\mathbf{1 8 c}$, respectively. On the other hand, the 11-substituted-pyrimido $\left[3^{\prime}, 2^{\prime}: 4,5\right]$ thieno[3,2:4,5]pyrimido[1,6-b][1,2,4]triazepines 21 a-21e were also obtained by the intramolecular cyclization of compound (10) with appropriate enaminone derivatives $19 \mathrm{a}-19 \mathrm{e}$ under the acidic condition.

\section{Introduction}

Pyrimidine and its derivatives are biologically important as antimicrobial [1, 2], antitumor [3], antihypertensive [4], and anti-inflammatory activities [5]. Among the derivatives of thieno[2,3-d]pyrimidines, substances have been observed that have antiviral, fungicidal, and insecticidal activity [6], antibacterial and antiplastic properties [7], antihypertensive [8] and anticonvulsant activity [9], and antihistaminic [10] action. Compounds with triazepine skeletons have attracted much attention as a result of their interesting biological properties $[11,12]$. On the other hand, condensed heterocyclic 1,2,4-triazepines were found to have salidiuretic and renal vasodilator, antioxidant, and analgesic and immunomodulating activities [13-15]. Further, the fusion of pyrimidine with triazepine moiety shows enhanced pharmacological effects as antiviral, antifungal [16], and antidiabetic [17] and also acts as inhibitors [18] in cancer chemotherapy.
Recently, it has been demonstrated that heterocycles attached to seven-membered rings show important biological activities [19-21]. Likewise, fused triazepine derivatives with a bridgehead nitrogen atom in the molecule exhibit interesting biological properties [22]. Various conventional methods for the synthesis of fused triazepines are exemplified in the literature [23, 24] using cycloaddition [25] and photochemical methods, but pyrimido $\left[3^{\prime}, 2^{\prime}: 4,5\right]$ thieno $[3,2: 4,5]$ pyrimido $[1,6-b][1,2,4]$ triazepines are the least investigated group among the fused triazepines. Moreover, no general method is ever reported for the synthesis of the title compounds starting from 7 amino-8-imino-pyrimido[3,2:4,5] thieno[2,3- $d$ ] pyrimidine (10). In view of these, it was considered of interest to synthesize some new triazoles and triazepine derivatives of structure fused to pyrimido[3,2:4,5] thieno[2,3- $d]$ pyrimidine (PTP) ring in the hope that they may be biologically active. In preceding papers [26-29], we have described the 
synthesis of some new pyrimido[2,3:4,3]pyrazolo[1,5-a]pyrimidines, 1,2,4-triazolo[1,5-a]pyrimidothieno[2,3-d]pyrimidine, and 1,3,4-oxadiazole-thieno[2,3- $d$ ] pyrimidines from 5-cyano-1,6-dihydro-4-methyl-2-phenyl-6-thioxopyrimidine 1 [26], respectively. In continuation of our studies [26-30], we report herein the synthesis of some new pyrimido $\left[3^{\prime}, 2^{\prime}: 4,5\right]$ thieno[3,2-e] $[1,2,4]$-triazolo[ 1,5 -c] pyrimidines and pyrimido $\left[3^{\prime}, 2^{\prime}: 4,5\right]$ thieno[3,2:4,5]pyrimido[1,6b] $[1,2,4]$ triazepine derivatives by making use of the key intermediate 7 -amino-8-imino-pyrimido $[3,2: 4,5]$ thieno $[2,3$ d]pyrimidine (10), easily obtained from the thioxopyrimidine 1. The substrate proved to be a versatile compound by virtue of its vicinal amino and imino functions, evaluating the reactivity in several cyclization reactions performed with the aim of obtaining new triazoles and triazepines with a conserved PTP core.

\section{Experimental}

All melting points are uncorrected and in ${ }^{\circ} \mathrm{C}$. IR spectra were recorded on a JASCO FTIR- 3 spectrometer $(\mathrm{KBr}) ;{ }^{1} \mathrm{H}$ NMR spectra were obtained on a Bruker AM-300 WB FINMR spectrometer, and chemical shifts are expressed in $\delta$ ppm using TMS as an internal standard. Electron impact mass spectra were obtained at $70 \mathrm{eV}$ using a Finnigan Mat TSQ-46C spectrometer. Microanalyses for $\mathrm{C}, \mathrm{H}$, and $\mathrm{N}$ were performed on a Perkin-Elmer 240 elemental analyzer. Commercially available reagents were purchased from Aldrich and used directly. Reactions were routinely monitored by thin layer chromatography (TLC (ethyl acetate and hexane (3:7))) on silica gel (precoated $\mathrm{F}_{245}$ Merck plates). Compounds 17a17c $[30,31]$ and 19a-19e [26] were prepared according to known procedures.

2.1. 5-Amino-6-cyano-4-methyl-2-phenyl-thieno[2,3-d] pyrimidine (3). A mixture of compound $\mathbf{1}(2.27 \mathrm{~g}, 10 \mathrm{mmol})$, potassium carbonate anhydrous $(2.76 \mathrm{~g}, 20 \mathrm{mmol})$, and chloroacetonitrile $2(0.64 \mathrm{~g}, 10 \mathrm{mmol})$ in DMF $(50 \mathrm{~mL})$ was stirred at room temperature for $4 \mathrm{~h}$ and then diluted with cold water $(50 \mathrm{~mL})$. The resulting solid product was collected by filtration, washed with water, and recrystallized from ethyl acetate/ethanol to give pale yellow needles. Yield $2.44 \mathrm{~g}(92 \%)$, mp 259 C; IR: $v$ 3511, $3359\left(\mathrm{NH}_{2}\right), 2204(\mathrm{CN}) \mathrm{cm}^{-1} ;{ }^{1} \mathrm{H}$ NMR (DMSO-d $\left.\mathrm{d}_{6}\right): \delta 2.60\left(3 \mathrm{H}, \mathrm{s}, \mathrm{CH}_{3}\right), 6.23\left(2 \mathrm{H}, \mathrm{br}, \mathrm{NH}_{2}\right)$, 8.55-8.53, 7.53-7.48 (5H, m, phenyl-H); MS: $266\left(\mathrm{M}^{+}, 100\right)$. Anal. Calcd. for $\mathrm{C}_{14} \mathrm{H}_{10} \mathrm{~N}_{4} \mathrm{~S}$ : C, 63.15; H, 3.75; N, 21.05 . Found: C, 63.23; H, 3.80; N, 21.12\%.

2.2. 6-Cyano-5-ethoxymethyleneamino-4-methyl-2-phenylthieno[2,3-d]pyrimidine (4). A solution of compound 3 $(2.66 \mathrm{~g}, 10 \mathrm{mmol})$ and triethyl orthoformate $(8 \mathrm{~mL})$ was refluxed in acetic anhydride $(15 \mathrm{~mL})$ for $24 \mathrm{~h}$. The reaction mixture was cooled. The resulting solid product was collected by filtration and recrystallized from ethanol to furnish $\mathbf{4}$ as pale yellow crystals. Yield $2.67 \mathrm{~g}(83 \%), \mathrm{mp} 150^{\circ} \mathrm{C}$; IR: $v 2215$ $(\mathrm{CN}), 1627(\mathrm{~N}=\mathrm{C}) \mathrm{cm}^{-1} ;{ }^{1} \mathrm{H}$ NMR $\left(\mathrm{CDCl}_{3}\right): \delta 1.48(3 \mathrm{H}, \mathrm{t}, J$ $\left.=1.42 \mathrm{~Hz}, \mathrm{CH}_{3}\right), 2.92\left(3 \mathrm{H}, \mathrm{s}, \mathrm{CH}_{3}\right), 4.50(2 \mathrm{H}, \mathrm{q}, J=2.12 \mathrm{~Hz}$,
$\left.\mathrm{OCH}_{2}\right), 8.09(1 \mathrm{H}, \mathrm{s}, \mathrm{N}=\mathrm{CH}), 8.55-8.53,7.52-7.50(5 \mathrm{H}, \mathrm{m}$, phenyl-H); MS: $322\left(\mathrm{M}^{+}, 100\right), 293(13), .277(32), 266(96)$, 250(4), 191(5), 163(33), 153(9), 104(18), 77(19). Anal. Calcd. for $\mathrm{C}_{17} \mathrm{H}_{14} \mathrm{~N}_{4} \mathrm{OS}$ : C, 63.35; H, 4.34; N, 17.39. Found: C, 63.33; H, 4.40; N, 17.41\%.

\subsection{7-Ethyl-8-imino-4-methyl-2-phenyl-7,8-dihydropyrimido} [3,2:4,5]thieno[2,3-d]pyrimidine (6a). To a solution of compound $4(0.322 \mathrm{~g}, 1 \mathrm{mmol})$ in ethanol $(10 \mathrm{~mL})$, ethylamine $(10 \mathrm{~mL})$ was added. The reaction mixture was refluxed for 4-5 h. After cooling, the precipitate was filtered and recrystallized from acetic acid/ethanol to furnish $\mathbf{6 a}$ as pale yellow crystals. Yield $0.29 \mathrm{~g}(92 \%), \mathrm{mp} 225^{\circ} \mathrm{C}$; IR: $v 3307(\mathrm{NH}), 1614$ $(\mathrm{C}=\mathrm{N}) \mathrm{cm}^{-1} ;{ }^{1} \mathrm{H}$ NMR $\left(\mathrm{CDCl}_{3}\right): \delta 1.47(3 \mathrm{H}, \mathrm{t}, J=1.47 \mathrm{~Hz}$, $\left.\mathrm{CH}_{3}\right), 3.13\left(3 \mathrm{H}, \mathrm{s}, \mathrm{CH}_{3}\right), 4.10\left(2 \mathrm{H}, \mathrm{q}, J=2.15 \mathrm{~Hz}, \mathrm{CH}_{2}\right)$, 8.57-8.55, 7.52-7.50 (5H, m, phenyl-H), $7.90(1 \mathrm{H}, \mathrm{s}, 6-\mathrm{H})$; MS: $321\left(\mathrm{M}^{+}, 100\right)$. Anal. Calcd. for $\mathrm{C}_{17} \mathrm{H}_{15} \mathrm{~N}_{5} \mathrm{~S}: \mathrm{C}, 63.55 ; \mathrm{H}$, 4.67; N, 21.80. Found: C, 63.65; H, 4.70; N, 21.97\%.

2.4. 2,7-Diphenyl-8-imino-4-methyl-7,8-dihydropyrimido [3, $2: 4,5]$ thieno [2,3-d]pyrimidine (6b). This compound was synthesized from compound $4(0.322 \mathrm{~g}, 1 \mathrm{mmol})$ and aniline $(0.092 \mathrm{~g}, 1 \mathrm{mmol})$ in a similar way to that described for the preparation of 6a. It was recrystallized from acetic acid/DMF to furnish $\mathbf{6 b}$ as pale yellow crystals. Yield $0.33 \mathrm{~g}(90 \%)$, mp $249^{\circ} \mathrm{C}$; IR: $v 3310(\mathrm{NH}), 1609(\mathrm{C}=\mathrm{N}) \mathrm{cm}^{-1} ;{ }^{1} \mathrm{H}$ NMR $\left(\mathrm{CF}_{3} \mathrm{COOD}\right): \delta 2.68\left(3 \mathrm{H}, \mathrm{s}, \mathrm{CH}_{3}\right), 8.87-8.85,8.33-8.01$ $(10 \mathrm{H}, \mathrm{m}$, phenyl-H), $9.22(1 \mathrm{H}, \mathrm{s}, 6-\mathrm{H}), 9.41(1 \mathrm{H}, \mathrm{br}, \mathrm{NH})$; MS: 369( $\left.\mathrm{M}^{+}, 100\right), 265(12), 184(11), 153(2), 136(2), 104(9)$, 77(21). Anal. Calcd. for $\mathrm{C}_{21} \mathrm{H}_{15} \mathrm{~N}_{5} \mathrm{~S}$ : C, 68.29; H, 4.06; N, 18.97. Found: C, $68.49 ; \mathrm{H}, 4.30 ; \mathrm{N}, 19.19 \%$.

2.5. 7-Formyl-8-imino-4-methyl-2-phenyl-7,8-dihydropyrimido [3,2:4,5]thieno[2,3-d]pyrimidine (7). A mixture of compound $4(0.322 \mathrm{~g}, 1 \mathrm{mmol})$ and formamide $(10 \mathrm{~mL})$ was refluxed for $1 \mathrm{~h}$. After cooling, the precipitate was filtered and recrystallized from acetic acid/ethanol to furnish 7 as brown crystals. Yield $0.31 \mathrm{~g}(97 \%), \mathrm{mp} 240^{\circ} \mathrm{C}$; IR: $\nu 3305(\mathrm{NH}), 1678$ (CO), $1616(\mathrm{C}=\mathrm{N}) \mathrm{cm}^{-1} ;{ }^{1} \mathrm{H}$ NMR $\left(\mathrm{DMSO}-\mathrm{d}_{6}\right): \delta 3.10(3 \mathrm{H}, \mathrm{s}$, $\left.\mathrm{CH}_{3}\right), 8.86(1 \mathrm{H}, \mathrm{s}, 6-\mathrm{H}), 8.45-8.44,7.54-7.47$ (5H, m, phenyl$\mathrm{H}), 8.33(1 \mathrm{H}, \mathrm{br}, \mathrm{NH}), 8.54(1 \mathrm{H}, \mathrm{s}, \mathrm{CHO}), \mathrm{MS}: 321\left(\mathrm{M}^{+}, 2\right)$, 293(100), 266(2), 190(22), 163(9), 136(2), 103(4), 77(2). Anal. Calcd. for $\mathrm{C}_{16} \mathrm{H}_{11} \mathrm{~N}_{5} \mathrm{OS}$ : C, 59.81; H, 3.42; N, 21.80. Found: C, $59.95 ; \mathrm{H}, 3.33 ; \mathrm{N}, 21.92 \%$.

2.6. 2, 9-Diphenyl-7-methyl-pyrimido $\left[3^{\prime}, 2^{\prime}: 4,5\right]$ thieno[3,2e][1,2,4]triazolo[1,5-c]pyrimidine (9a). A mixture of compound $4(0.322 \mathrm{~g}, 1 \mathrm{mmol})$ and benzoylhydrazine 8a $(0.136 \mathrm{~g}, 1 \mathrm{mmol})$ in 2-methoxyethanol $(10 \mathrm{~mL})$ was refluxed for $7 \mathrm{~h}$. After cooling, the resulting solid product was collected by filtration and washed with water, and the crude product was recrystallized from ethanol/glacial acetic acid to furnish $9 \mathrm{a}$ as white crystals. Yield $0.34 \mathrm{~g}(87 \%), \mathrm{mp}$ $306^{\circ}$ C; IR: $v 1603(\mathrm{C}=\mathrm{N}) \mathrm{cm}^{-1}$; ${ }^{1} \mathrm{H}$ NMR $\left(\mathrm{CF}_{3} \mathrm{COOD}\right): \delta$ $3.42\left(3 \mathrm{H}, \mathrm{s}, \mathrm{CH}_{3}\right), 8.43-8.40,7.88-7.76(10 \mathrm{H}, \mathrm{m}$, phenyl-H), 9.93 (1H, s, 5-H); MS: $394\left(\mathrm{M}^{+}, 100\right), 317(2), 290(20), 262(3)$, 197(10), 153(3), 118(10), 105(20), 77(24). Anal. Calcd. for 
$\mathrm{C}_{22} \mathrm{H}_{14} \mathrm{~N}_{6} \mathrm{~S}: \mathrm{C}, 67.00 ; \mathrm{H}, 3.55 ; \mathrm{N}, 21.31$. Found: C, 67.23; H, $3.42 ; \mathrm{N}, 21.45 \%$.

2.7. 2-(4-Pyridyl)-7-methyl-9-phenyl-pyrimido $\left[3^{\prime}, 2^{\prime}: 4,5\right]$ thieno[3,2-e][1,2,4]triazolo[1,5-c]pyrimidine (9b). This compound was synthesized from compound $4(0.322 \mathrm{~g}, 1 \mathrm{mmol})$ and isonicotinic acid hydrazide $(0.137 \mathrm{~g}, 1 \mathrm{mmol})$ in a similar way to that described for the preparation of 9a. It was recrystallized from ethanol/DMF to furnish $\mathbf{9 b}$ as pale yellow crystals. Yield $0.32 \mathrm{~g}(83 \%), \mathrm{mp}>340^{\circ} \mathrm{C}$; IR: $v 1607(\mathrm{C}=\mathrm{N})$ $\mathrm{cm}^{-1} ;{ }^{1} \mathrm{H}$ NMR $\left(\mathrm{CDCl}_{3}\right): \delta 3.73\left(3 \mathrm{H}, \mathrm{s}, \mathrm{CH}_{3}\right), 7.87-7.82$, 7.74-7.71 (5H, m, phenyl-H), $8.40(2 \mathrm{H}, \mathrm{d}, J=1.00 \mathrm{~Hz}, 3,5-$ $\mathrm{H}$ of pyridyl), $8.85(2 \mathrm{H}, \mathrm{d}, J=1.00 \mathrm{~Hz}, 4,6-\mathrm{H}$ of pyridyl), $9.83(1 \mathrm{H}, \mathrm{s}, 5-\mathrm{H})$; MS: 395( $\left.\mathrm{M}^{+}, 100\right), 317(2), 291(20), 267(4)$, 236(1), 197(10), 190(2), 103(4), 77(8). Anal. Calcd. for $\mathrm{C}_{21} \mathrm{H}_{13} \mathrm{~N}_{7} \mathrm{~S}$ : C, 63.79; H, 3.29; N, 24.81. Found: C, 63.98; H, $3.42 ; \mathrm{N}, 24.99 \%$.

2.8. 7-Amino-8-imino-4-methyl-2-phenyl-7,8-dihydropyrimido[3,2:4,5]thieno[2,3-d]pyrimidine (10). A mixture of compound $4(0.322 \mathrm{~g}, 1 \mathrm{mmol})$ and hydrazine hydrate $(8 \mathrm{~mL}$, $80 \%)$ in ethanol $(20 \mathrm{~mL})$ was refluxed for $24 \mathrm{~h}$. After cooling, the resulting solid product was collected by filtration and washed with water, and the crude product was recrystallized from ethanol/DMF to furnish (10) as pale yellow crystals. Yield $0.27 \mathrm{~g}(89 \%), \mathrm{mp} 285^{\circ} \mathrm{C}$; IR: $v$ 3315, 3247 $\left(\mathrm{NH}_{2}, \mathrm{NH}\right), 1603(\mathrm{C}=\mathrm{N}) \mathrm{cm}^{-1} ;{ }^{1} \mathrm{H}$ NMR $\left(\mathrm{CF}_{3} \mathrm{COOD}\right): \delta$ $3.21\left(3 \mathrm{H}, \mathrm{s}, \mathrm{CH}_{3}\right), 5.13\left(2 \mathrm{H}, \mathrm{s}, \mathrm{NH}_{2}\right), 8.11-8.07,7.51-7.37$ $(5 \mathrm{H}, \mathrm{m}$, phenyl-H), $8.69(1 \mathrm{H}, \mathrm{s}, 6-\mathrm{H}), 9.23(\mathrm{H}, \mathrm{s}, \mathrm{NH})$; MS: 308( $\left.\mathrm{M}^{+}, 100\right), 292(2), 278(38), 251(18), 225(2), 188(4)$, 176(10), 148(6), 120(4), 104(19), 77(16). Anal. Calcd. for $\mathrm{C}_{15} \mathrm{H}_{12} \mathrm{~N}_{6} \mathrm{~S}: \mathrm{C}, 58.44 ; \mathrm{H}, 3.89 ; \mathrm{N}, 27.27$. Found: C, 58.66; H, $3.82 ; \mathrm{N}, 27.36 \%$.

2.9. 8-(Acetylamino)-7-diacetylamino-4-methyl-2-phenyl-pyrimido[3,2:4,5] thieno[2,3-d]pyrimidine (11). A mixture of compound (10) $(0.308 \mathrm{~g}, 1 \mathrm{mmol})$ and acetic anhydride $(10 \mathrm{~mL})$ was refluxed for $2 \mathrm{~h}$. After cooling, the precipitate was filtered and recrystallized from acetic acid/ethanol to furnish (11) as orange crystals. Yield $0.41 \mathrm{~g}(96 \%), \mathrm{mp} 236^{\circ} \mathrm{C}$; IR: $v 1718(\mathrm{CO}), 1639(\mathrm{C}=\mathrm{N}) \mathrm{cm}^{-1} ;{ }^{1} \mathrm{H}$ NMR $\left(\mathrm{CF}_{3} \mathrm{COOD}\right): \delta$ $2.32\left(3 \mathrm{H}, \mathrm{s}, \mathrm{COCH}_{3}\right), 2.56\left(6 \mathrm{H}, \mathrm{s},\left(\mathrm{COCH}_{3}\right)_{2}\right), 2.79(3 \mathrm{H}, \mathrm{s}$, $\left.\mathrm{CH}_{3}\right), 8.47-8.44,7.97-7.78(5 \mathrm{H}, \mathrm{m}$, phenyl-H), $9.15(1 \mathrm{H}, \mathrm{s}$, 6-H); MS: 434( $\left.\mathrm{M}^{+}, 20\right), 393(40), 350(93), 308(100), 280(34)$, 251(12), 191(2), 176(6), 148(5), 120(4), 103(3). Anal. Calcd. for $\mathrm{C}_{21} \mathrm{H}_{18} \mathrm{~N}_{6} \mathrm{O}_{3} \mathrm{~S}$ : C, 58.06; H, 4.14; N, 19.35. Found: C, $58.26 ; \mathrm{H}, 4.04 ; \mathrm{N}, 19.48 \%$.

2.10. 2,7-Dimethyl-9-phenyl-pyrimido $\left[3^{\prime}, 2^{\prime}: 4,5\right]$ thieno $[3,2-e]$ [1,2,4]triazolo[1,5-c]pyrimidine (12). A mixture of compound $(\mathbf{1 0})(0.308 \mathrm{~g}, 1 \mathrm{mmol})$ and glacial acetic acid $(10 \mathrm{~mL})$ was refluxed for $2 \mathrm{~h}$. After cooling, the precipitate was filtered and recrystallized from acetic acid/ethanol to furnish (12) as pale brown crystals. Yield $0.31 \mathrm{~g}(93 \%), \mathrm{mp} 271^{\circ} \mathrm{C}$; IR: $v$ $1615(\mathrm{C}=\mathrm{N}) \mathrm{cm}^{-1} ;{ }^{1} \mathrm{H}$ NMR (DMSO- $\left.\mathrm{d}_{6}\right): \delta 2.06\left(3 \mathrm{H}, \mathrm{s}, \mathrm{CH}_{3}\right)$, $3.17\left(3 \mathrm{H}, \mathrm{s}, \mathrm{CH}_{3}\right), 8.51-8.49,7.58-7.53(5 \mathrm{H}, \mathrm{m}$, phenyl$\mathrm{H}), 8.72(1 \mathrm{H}, \mathrm{s}, 5-\mathrm{H})$; MS: 332( $\left.\mathrm{M}^{+}, 20\right), 318(6), 293(100)$,
278(11), 267(1), 229(5), 190(24), 163(9), 103(4). Anal. Calcd. for $\mathrm{C}_{17} \mathrm{H}_{12} \mathrm{~N}_{6} \mathrm{~S}: \mathrm{C}, 61.44 ; \mathrm{H}, 3.61 ; \mathrm{N}, 25.30$. Found: $\mathrm{C}, 61.69$; $\mathrm{H}, 3.42$; N, $25.55 \%$.

2.11. 7-(4-Dimethylaminophenyl)azo-8-imino-4-methyl-2phenyl-7,8-dihydropyrimido[3,2:4,5] thieno[2,3-d]pyrimidine (13). A mixture of compound (10) $(0.308 \mathrm{~g}, 1 \mathrm{mmol})$ and $\mathrm{N}, \mathrm{N}$-dimethyl-4-nitrosoaniline $(0.15 \mathrm{~g}, 1 \mathrm{mmol})$ in glacial acetic acid $(10 \mathrm{~mL})$ was stirred at $70-80^{\circ} \mathrm{C}$ for $2 \mathrm{~h}$. The reaction mixture was cooled and poured into ice-water; the precipitate was filtered and recrystallized from acetic acid/ethanol to furnish $\mathbf{1 3}$ as brown crystals. Yield $0.28 \mathrm{~g}$ (64\%), mp 247 C; IR: $v 3305(\mathrm{NH}), 1629(\mathrm{C}=\mathrm{N}) \mathrm{cm}^{-1}$; ${ }^{1} \mathrm{H}$ NMR ( $\left.\mathrm{CF}_{3} \mathrm{COOD}\right): \delta 3.51\left(6 \mathrm{H}, \mathrm{s}, \mathrm{N}\left(\mathrm{CH}_{3}\right)_{2}\right), 3.61(3 \mathrm{H}, \mathrm{s}$, $\left.\mathrm{CH}_{3}\right), 8.34-8.32,7.86-7.67(9 \mathrm{H}, \mathrm{m}$, phenyl-H), $8.66(1 \mathrm{H}$, s, 6-H); MS: 412( $\left.\mathrm{M}^{+} 10\right), 397(3), 347(3), 332(8), 319(13)$, 308(10), 293(100), 278(9), 267(8), 215(5), 191(19), 163(10), 136(11), 121(8), 104(8), 77(12). Anal. Calcd. for $\mathrm{C}_{23} \mathrm{H}_{20} \mathrm{~N}_{8} \mathrm{~S}$ : C, 62.72; H, 4.54; N, 25.45. Found: C, 62.86; H, 4.64; N, $25.68 \%$.

2.12. General Procedures for the Preparation of 2-(substitutedamino)-7-methyl-9-phenyl-pyrimido $\left[3^{\prime}, 2^{\prime}: 4,5\right]$ thieno[3,2-

e][1,2,4]triazolo[1,5-c]pyrimidines $(\mathbf{1 5 a}-\mathbf{1 5 g})$. A mixture of compound (10) $(0.308 \mathrm{~g}, 1 \mathrm{mmol})$ and appropriate isothiocyanates 14a-14g (1 mmol) in pyridine $(10 \mathrm{~mL})$ was refluxed for $7-8 \mathrm{~h}$. The reaction mixture was cooled and poured into ice-water the precipitate was filtered and recrystallized from DMF/ethanol to furnish 15a-15g.

2.13. 2-(Methylamino)-7-methyl-9-phenyl-pyrimido $\left[3^{\prime}, 2^{\prime}: 4\right.$, 5] thieno[3,2-e][1,2,4]triazolo[1,5-c]pyrimidine (15a): Yellowish Brown Crystals. Yield 0.19g (55\%), mp $286^{\circ} \mathrm{C}$; IR: $v 3309(\mathrm{NH}), 1612(\mathrm{C}=\mathrm{N}) \mathrm{cm}^{-1} ;{ }^{1} \mathrm{H}$ NMR $\left(\mathrm{CF}_{3} \mathrm{COOD}\right): \delta$ $3.35\left(3 \mathrm{H}, \mathrm{s}, \mathrm{CH}_{3}\right), 3.81\left(3 \mathrm{H}, \mathrm{s}, \mathrm{CH}_{3}\right), 8.53-8.45,7.99-7.81$ $(5 \mathrm{H}, \mathrm{m}$, phenyl-H), $9.48(1 \mathrm{H}, \mathrm{br}, \mathrm{NH}), 9.87(1 \mathrm{H}, \mathrm{s}, 5-\mathrm{H})$; MS: 347( $\left.\mathrm{M}^{+}, 31\right), 332(10), 304(3), 293(12), 278(7), 174(1)$, 163(9), 79(4). Anal. Calcd. for $\mathrm{C}_{17} \mathrm{H}_{13} \mathrm{~N}_{7} \mathrm{~S}$ : C, 58.78; H, 3.74; N, 28.24. Found: C, 58.89; H, 3.82; N, 28.45\%.

2.14. 2-(Ethylamino)-7-methyl-9-phenyl-pyrimido $\left[3^{\prime}, 2^{\prime}: 4\right.$, 5]thieno[3,2-e][1,2,4]-triazolo[1,5-c]pyrimidine (15b). Pale greenish yellow crystals. Yield $0.10 \mathrm{~g}(28 \%), \mathrm{mp} 327^{\circ} \mathrm{C}$; IR: $v$ $3303(\mathrm{NH}), 1614(\mathrm{C}=\mathrm{N}) \mathrm{cm}^{-1}{ }^{1}{ }^{1} \mathrm{H}$ NMR $\left(\mathrm{CF}_{3} \mathrm{COOD}\right): \delta 1.92$ $\left(3 \mathrm{H}, \mathrm{t}, J=1.50 \mathrm{~Hz}, \mathrm{CH}_{3}\right), 3.81\left(3 \mathrm{H}, \mathrm{s}, \mathrm{CH}_{3}\right), 4.92(2 \mathrm{H}, \mathrm{q}, J=$ $\left.1.00 \mathrm{~Hz}, \mathrm{CH}_{2}\right), 8.55-8.49,8.00-7.81(5 \mathrm{H}, \mathrm{m}$, phenyl-H), 9.15 $(1 \mathrm{H}, \mathrm{br}, \mathrm{NH}), 9.88(1 \mathrm{H}, \mathrm{s}, 5-\mathrm{H})$; MS: $361\left(\mathrm{M}^{+}, 41\right), 350(84)$, 347(100), 332(8), 319(22), 304(4), 293(12), 278(16), 265(8), 215(18), 174(20), 147(9), 103(38), 77(10). Anal. Calcd. for $\mathrm{C}_{18} \mathrm{H}_{15} \mathrm{~N}_{7} \mathrm{~S}$ : C, 59.83; H, 4.15; N, 27.14. Found: C, 59.99; H, $4.35 ; \mathrm{N}, 27.26 \%$.

2.15. 2-(Phenylamino)-7-methyl-9-phenyl-pyrimido $\left[3^{\prime}, 2^{\prime}: 4\right.$, 5] thieno[3,2-e][1,2,4]triazolo[1,5-c]pyrimidine (15c): Greenish Yellow Crystals. Yield $0.20 \mathrm{~g}$ (49\%), mp 295 ${ }^{\circ}$; IR: $v 3311(\mathrm{NH}), 1605(\mathrm{C}=\mathrm{N}) \mathrm{cm}^{-1} ;{ }^{1} \mathrm{H}$ NMR $\left(\mathrm{CF}_{3} \mathrm{COOD}\right)$ : 
$\delta 3.78\left(3 \mathrm{H}, \mathrm{s}, \mathrm{CH}_{3}\right), 8.46-8.45,7.92-7.77(10 \mathrm{H}, \mathrm{m}$, phenyl$\mathrm{H}), 9.79$ (1H, s, 5-H); MS: 409( $\left.\mathrm{M}^{+}, 21\right), 350(100), 332(8)$, 304(15), 293(16), 278(8), 247(5), 201(10), 174(8), 147(3), 103(10), 77(4). Anal. Calcd. for $\mathrm{C}_{22} \mathrm{H}_{15} \mathrm{~N}_{7} \mathrm{~S}$ : C, 64.54; $\mathrm{H}$, 3.66; N, 23.96. Found: C, 64.69; H, 3.88; N, 24.11\%.

2.16. 2-(4-Chlorophenylamino)-7-methyl-9-phenyl-pyrimido $\left[3^{\prime}, 2^{\prime}: 4,5\right]$ thieno[3,2-e][1,2,4]triazolo[1,5-c]pyrimidine (15d) Pale Greenish Yellow Crystals. Yield $0.27 \mathrm{~g}(62 \%), \mathrm{mp} 266^{\circ} \mathrm{C}$; IR: $v 3314(\mathrm{NH}), 1612(\mathrm{C}=\mathrm{N}) \mathrm{cm}^{-1}{ }^{1} \mathrm{H}$ NMR $\left(\mathrm{CF}_{3} \mathrm{COOD}\right)$ : $\delta 2.45\left(3 \mathrm{H}, \mathrm{s}, \mathrm{CH}_{3}\right), 7.68(2 \mathrm{H}, \mathrm{d}, J=1.00 \mathrm{~Hz}, 3,5-\mathrm{H}$ of phenyl-H), $7.78(2 \mathrm{H}, \mathrm{d}, J=1.00 \mathrm{~Hz}, 2,6-\mathrm{H}$ of phenyl-H), 8.65-8.64, 8.09-7.97 (5H, m, phenyl-H), 9.98 (1H, s, 5$\mathrm{H})$; MS: 443.5( $\left.\mathrm{M}^{+}, 21\right), 407(2), 392(14), 350(100), 332(4)$, 319(5), 304(12), 293(30), 278(11), 255(13), 247(5), 213(16), 174(16), 147(40), 103(20), 91(31), 71(33). Anal. Calcd. for $\mathrm{C}_{22} \mathrm{H}_{14} \mathrm{ClN}_{7} \mathrm{~S}: \mathrm{C}, 59.52 ; \mathrm{H}, 3.15 ; \mathrm{N}, 22.09$. Found: C, 59.69; H, 3.28; N, 22.21\%.

2.17. 2-(Benzylamino)-7-methyl-9-phenyl-pyrimido $\left[3^{\prime}, 2^{\prime}: 4\right.$, 5]thieno[3,2-e][1,2,4]triazolo[1,5-c]pyrimidine (15e): Yellow Crystals. Yield $0.20 \mathrm{~g}(47 \%)$, mp $306^{\circ} \mathrm{C}$; IR: $v 3314(\mathrm{NH})$, $1601(\mathrm{C}=\mathrm{N}) \mathrm{cm}^{-1} ;{ }^{1} \mathrm{H} \mathrm{NMR}\left(\mathrm{CF}_{3} \mathrm{COOD}\right): \delta 2.60(3 \mathrm{H}, \mathrm{s}$, $\left.\mathrm{CH}_{3}\right), 4.15\left(2 \mathrm{H}, \mathrm{s}, \mathrm{CH}_{2}\right), 8.80-8.79,8.29-7.83(10 \mathrm{H}, \mathrm{m}$, phenyl-H), 8.79 (1H, s, 5-H); MS: 422( $\left.\mathrm{M}^{+}, 10\right), 350(100)$, 332(2), 308(20), 304(8), 293(9), 278(12), 265(6), 247(2), 201(4), 174(6), 153(4), 103(28), 91(14), 77(7). Anal. Calcd. for $\mathrm{C}_{23} \mathrm{H}_{16} \mathrm{~N}_{7} \mathrm{~S}$ : C, 65.40; H, 3.79; N, 23.22. Found: C, 65.49; H, 3.88; N, 23.36\%.

2.18. 2-(2-Mthylphenylamino)-7-methyl-9-phenyl-pyrimido $\left[3^{\prime}, 2^{\prime}: 4,5\right]$ thieno[3,2-e][1,2,4] triazolo[1,5-c]pyrimidine (15f): Greenish Yellow Crystals. Yield $0.18 \mathrm{~g}(43 \%), \mathrm{mp} 285^{\circ} \mathrm{C}$; IR: v $3308(\mathrm{NH}), 1608(\mathrm{C}=\mathrm{N}) \mathrm{cm}^{-1} ;{ }^{1} \mathrm{H}$ NMR ( $\left.\mathrm{CF}_{3} \mathrm{COOD}\right): \delta$ $2.47\left(3 \mathrm{H}, \mathrm{s}, \mathrm{CH}_{3}\right), 2.54\left(3 \mathrm{H}, \mathrm{s}, \mathrm{CH}_{3}\right), 8.78-8.45,8.24-7.79$ (9H, m, phenyl-H), $9.78(1 \mathrm{H}, \mathrm{s}, 5-\mathrm{H})$; MS: 423( $\left.\mathrm{M}^{+}, 6\right)$, 350(100), 332(12), 318(20), 304(8), 293(68), 278(7), 265(8), 247(3), 215(6), 201(5), 190(16), 174(6), 147(3), 103(6), 77(4). Anal. Calcd. for $\mathrm{C}_{23} \mathrm{H}_{17} \mathrm{~N}_{7} \mathrm{~S}$ : C, 65.24; H, 4.01; N, 23.16. Found: C, 65.36; H, 3.95; N, 23.25\%.

2.19. 2-(1-Naphthylamino)-7-methyl-9-phenyl-pyrimido[3', $\left.2^{\prime}: 4,5\right]$ thieno[3,2-e][1,2,4] triazolo[1,5-c]pyrimidine $\quad(\mathbf{1 5 g})$ : Greenish Yellow Crystals. Yield $0.23 \mathrm{~g}$ (51\%), mp 282 C; IR: v $3309(\mathrm{NH}), 1613(\mathrm{C}=\mathrm{N}) \mathrm{cm}^{-1} ;{ }^{1} \mathrm{H}$ NMR $\left(\mathrm{CF}_{3} \mathrm{COOD}\right): \delta$ $2.41\left(3 \mathrm{H}, \mathrm{s}, \mathrm{CH}_{3}\right), 8.58-8.55,8.32-7.89(12 \mathrm{H}, \mathrm{m}$, phenyl-H and naphthyl-H), $9.89(1 \mathrm{H}, \mathrm{s}, 5-\mathrm{H})$; MS: 459( $\left.\mathrm{M}^{+}, 7\right), 396(4)$, 350(100), 332(18), 318(18), 304(11), 293(61), 278(6), 265(7), 247(5), 201(7), 174(8), 163(10), 147(6), 103(10), 77(7). Anal. Calcd. for $\mathrm{C}_{26} \mathrm{H}_{17} \mathrm{~N}_{7} \mathrm{~S}$ : C, 67.97; H, 3.70; N, 21.35. Found: C, $68.11 ; \mathrm{H}, 3.88 ; \mathrm{N}, 21.47 \%$.

2.20. 4-Methyl-2-phenyl-8H-9,10,11-trihydropyrimido $\left[3^{\prime}, 2^{\prime}\right.$ : 4,5] thieno[3,2:4, 5]pyrimido[1,6-b][1,2,4]triazepine (16). There was a mixture of compound (10) $(0.308 \mathrm{~g}, 1 \mathrm{mmol})$, potassium carbonate anhydrous $(3.036 \mathrm{~g}, 2.2 \mathrm{mmol})$, and 1,3-dibromopropane $(0.201 \mathrm{~g}, 1 \mathrm{mmol})$ in DMF $(30 \mathrm{~mL})$. The reaction mixture was stirred at $60^{\circ} \mathrm{C}$ for $9 \mathrm{~h}$. After cooling, the reaction mixture was poured into ice-water $(50 \mathrm{~mL})$ and neutralized with $10 \%$ hydrochloric acid. The solid formed was collected by filtration, washed with water, and recrystallized from acetic acid/ethanol to give reddish brown crystals. Yield $0.29 \mathrm{~g}(83 \%), \mathrm{mp} 175^{\circ} \mathrm{C}$; IR: $v 3308(\mathrm{NH})$, $1611(\mathrm{C}=\mathrm{N}) \mathrm{cm}^{-1} ;{ }^{1} \mathrm{H}$ NMR (CF3COOD): $\delta 2.32(2 \mathrm{H}, \mathrm{m}$, $\left.10-\mathrm{CH}_{2}\right), 2.92\left(3 \mathrm{H}, \mathrm{s}, \mathrm{CH}_{3}\right), 3.42\left(2 \mathrm{H}, \mathrm{t}, J=1.60 \mathrm{~Hz}, 11-\mathrm{CH}_{2}\right)$, $4.42\left(2 \mathrm{H}, \mathrm{t}, J=1.70 \mathrm{~Hz}, 9-\mathrm{CH}_{2}\right), 8.45-8.33,7.84-7.81(5 \mathrm{H}$, m, phenyl-H), $9.84(1 \mathrm{H}, \mathrm{s}, 6-\mathrm{H}), 10.12$ (1H, br, NH); MS: 348 $\left(\mathrm{M}^{+}, 11\right), 333(13), 318(89), 308(8), 293(39), 218(100)$, 263(3), 251(8), 215(9), 190(14), 175(38), 163(9), 121(10), 104(21), 77(22). Anal. Calcd. for $\mathrm{C}_{18} \mathrm{H}_{16} \mathrm{~N}_{6} \mathrm{~S}$ : C, 62.06; $\mathrm{H}$, 4.59 ; N, 24.13. Found: C, 62.19; H, 4.65; N, 24.26\%.

2.21. General Procedures for the Preparation of 9substituted-4-methyl-2-phenyl-10,11-dihydro-pyrimido[3', $2^{\prime}$ : 4,5] thieno[3,2:4,5]pyrimido[1,6-b][1,2,4]triazepines (18a18c). A mixture of compound (10) $(0.308 \mathrm{~g}, 1 \mathrm{mmol})$, Mannish base 17a-17c (N,N-dimethyl-2-benzolethylamine hydrochloride 17a, N,N-dimethyl-2-furoylethyl- amine hydrochloride $\mathbf{1 7 b}$, and N,N-dimethyl-2-thenoylethylamine hydrochloride $17 \mathrm{c})(1 \mathrm{mmol})$, and anhydrous potassium carbonate $(0.304 \mathrm{~g}, 2.2 \mathrm{mmol})$ was refluxed in DMF $(10 \mathrm{~mL})$ for $8 \mathrm{~h}$. After cooling, solid formed was collected by filtration and recrystallized from chloroform/ethanol.

2.22. 2,9-Diphenyl-4-methyl-10,11-dihydropyrimido $\left[3^{\prime}, 2^{\prime}\right.$ : 4,5] thieno[3,2:4,5]pyrimido[1,6-b][1,2,4]triazepine (18a): Pale Brown Crystals. Yield $0.35 \mathrm{~g}$ (83\%), mp $172^{\circ} \mathrm{C}$; IR: $v$ $1609(\mathrm{C}=\mathrm{N}) \mathrm{cm}^{-1} ;{ }^{1} \mathrm{H}$ NMR $\left(\mathrm{CDCl}_{3}\right): \delta 3.11\left(3 \mathrm{H}, \mathrm{s}, \mathrm{CH}_{3}\right)$, $3.26\left(2 \mathrm{H}, \mathrm{t}, J=1.00 \mathrm{~Hz}, 10-\mathrm{CH}_{2}\right), 4.21(2 \mathrm{H}, \mathrm{t}, J=1.00 \mathrm{~Hz}$, 11- $\left.\mathrm{CH}_{2}\right), 8.54-8.51,7.57-7.41(10 \mathrm{H}, \mathrm{m}$, phenyl- $\mathrm{H}), 8.72(1 \mathrm{H}$, s, 6-H); MS: 422( $\left.\mathrm{M}^{+}, 38\right), 394(5), 345(4), 332(13), 318(96)$, 293(100), 278(36), 250(7), 214(10), 190(39), 163(22), 159(9), 115(10), 103(39), 77(61). Anal. Calcd. for $\mathrm{C}_{24} \mathrm{H}_{18} \mathrm{~N}_{6} \mathrm{~S}$ : C, 68.24; H, 4.26; N, 19.90. Found: C, 68.49; H, 4.45; N, 20.15\%.

2.23. 9-(2-Furyl)-4-methyl-2-phenyl-10,11-dihydropyrimido[3' $\left.2^{\prime}: 4,5\right]$ thieno[3,2:4,5]pyrimido[1,6-b][1,2,4]triazepine (18b): Brown Crystals. Yield $0.32 \mathrm{~g}(79 \%)$, mp $162^{\circ} \mathrm{C}$; IR: $v$ $1615(\mathrm{C}=\mathrm{N}) \mathrm{cm}^{-1} ;{ }^{1} \mathrm{H}$ NMR $\left(\mathrm{CDCl}_{3}\right): \delta 3.16\left(3 \mathrm{H}, \mathrm{s}, \mathrm{CH}_{3}\right)$, $3.22\left(2 \mathrm{H}, \mathrm{t}, J=1.00 \mathrm{~Hz}, 10-\mathrm{CH}_{2}\right), 4.19(2 \mathrm{H}, \mathrm{t}, J=1.00 \mathrm{~Hz}$, $\left.11-\mathrm{CH}_{2}\right), 6.84(1 \mathrm{H}, \mathrm{dd}, J=1.00,1.00 \mathrm{~Hz}, 4-\mathrm{H}$ of furyl $)$, $7.59(1 \mathrm{H}, \mathrm{d}, J=1.00 \mathrm{~Hz}, 3-\mathrm{H}$ of furyl $), 8.49-8.44,7.54-7.49$ $(5 \mathrm{H}, \mathrm{m}$, phenyl-H), $8.54(1 \mathrm{H}, \mathrm{d}, J=1.0 \mathrm{~Hz}, 5-\mathrm{H}$ of furyl), 8.71 (1H, s, 6-H); MS: 412( $\left.\mathrm{M}^{+}, 20\right), 384(5), 345(4), 332(3)$, 318(50), 293(100), 278(26), 250(4), 215(8), 190(39), 163(21), 153(7), 120(7), 103(28), 95(29), 77(30), 51(9). Anal. Calcd. for $\mathrm{C}_{22} \mathrm{H}_{16} \mathrm{~N}_{6} \mathrm{OS}$ : C, 64.07; $\mathrm{H}, 3.88 ; \mathrm{N}, 20.38$. Found: C, 64.19; H, 3.98; N, 20.45\%.

2.24. 9-(2-Thienyl)-4-methyl-2-phenyl-10,11-dihydropyrimido $\left[3^{\prime}, 2^{\prime}: 4,5\right]$ thieno $[3,2: 4,5]$ pyrimido $[1,6-b][1,2,4]$ triazepine (18c): Pale Yellow Crystals. Yield $0.35 \mathrm{~g}(82 \%), \mathrm{mp} 154^{\circ} \mathrm{C}$; IR: $v 1621(\mathrm{C}=\mathrm{N}) \mathrm{cm}^{-1} ;{ }^{1} \mathrm{H} \mathrm{NMR}\left(\mathrm{CDCl}_{3}\right): \delta 3.16\left(3 \mathrm{H}, \mathrm{s}, \mathrm{CH}_{3}\right)$, $3.28\left(2 \mathrm{H}, \mathrm{t}, J=1.00 \mathrm{~Hz}, 10-\mathrm{CH}_{2}\right), 4.23(2 \mathrm{H}, \mathrm{t}, J=1.00 \mathrm{~Hz}$, 
$\left.11-\mathrm{CH}_{2}\right), 7.08(1 \mathrm{H}, \mathrm{dd}, J=1.00,1.00 \mathrm{~Hz}, 4-\mathrm{H}$ of thienyl), $8.57-8.40,7.54-7.42(6 \mathrm{H}, \mathrm{m}, 3-\mathrm{H}$ of thienyl and phenyl- $\mathrm{H})$, $8.62(1 \mathrm{H}, \mathrm{d}, J=1.00 \mathrm{~Hz}, 5-\mathrm{H}$ of thienyl), $8.73(1 \mathrm{H}, \mathrm{s}, 6-\mathrm{H})$; MS: 428( $\left.\mathrm{M}^{+}, 10\right), 400(5), 345(2), 332(6), 318(19), 293(100)$, 278(22), 250(4), 215(5), 190(40), 163(23), 153(9), 110(45), 103(32), 77(30), 51(10). Anal. Calcd. for $\mathrm{C}_{22} \mathrm{H}_{16} \mathrm{~N}_{6} \mathrm{~S}_{2}$ : C, $61.68 ; \mathrm{H}, 3.73 ; \mathrm{N}, 19.62$. Found: C, 61.78; H, 3.88; N, 19.80\%.

2.25. 7-[1-(4-Pyridyl)prop-2-enone-3-yl]amino-8-imino-4methyl-2-phenyl-7,8-dihydropyrimido [3,2:4,5] thieno[2,3-

d]pyrimidine (20). Method A: a mixture of compound (10) $(0.308 \mathrm{~g}, 1 \mathrm{mmol})$ and 3-dimethylamino-1-(4-pyridyl)prop2 -enone $19 \mathrm{e}(0.176 \mathrm{~g}, 1 \mathrm{mmol})$ in glacial acetic acid $(10 \mathrm{~mL})$ was stirred at $80^{\circ} \mathrm{C}$ for $7 \mathrm{~h}$. The reaction mixture was cooled. The resulting solid product was collected by filtration and recrystallized from DMF/ethanol to give brownish yellow crystals. Yield $0.26 \mathrm{~g}(60 \%), \mathrm{mp} 207^{\circ} \mathrm{C}$; IR: $v 3215(\mathrm{NH})$, $1668(\mathrm{C}=\mathrm{O}), 1632(\mathrm{C}=\mathrm{N}) \mathrm{cm}^{-1} ;{ }^{1} \mathrm{H}$ NMR $\left(\mathrm{CF}_{3} \mathrm{COOD}\right): \delta$ $2.59\left(3 \mathrm{H}, \mathrm{s}, \mathrm{CH}_{3}\right), 4.00(1 \mathrm{H}, \mathrm{d}, J=1.05 \mathrm{~Hz}, \mathrm{COCH}=), 8.68$ $(2 \mathrm{H}, \mathrm{d}, J=1.00 \mathrm{~Hz}, 3,5-\mathrm{H}$ of pyridyl), 8.78-8.74, 8.26-8.07 $(6 \mathrm{H}, \mathrm{m},-\mathrm{NCH}=$ and phenyl-H), $9.27(2 \mathrm{H}, \mathrm{d}, J=1.00 \mathrm{~Hz}$, 2,6- $\mathrm{H}$ of pyridyl), $9.53(1 \mathrm{H}, \mathrm{s}, 6-\mathrm{H})$; MS: $439\left(\mathrm{M}^{+}, 20\right)$, $421(100), 394(14), 367(29), 333(20), 319(88), 308(58)$, 293(29), 278(27), 215(9), 210(16), 174(15), 121(10), 104(22), 77(23), 51(14). Anal. Calcd. for $\mathrm{C}_{23} \mathrm{H}_{17} \mathrm{~N}_{7} \mathrm{OS}$ : C, 62.87; H, 3.87; N, 22.32. Found: C, 62.88; H, 3.98; N, 22.47\%. Method B: a mixture of compound (10) $(0.308 \mathrm{~g}, 1 \mathrm{mmol})$ and $19 \mathrm{e}(0.176 \mathrm{~g}, 1 \mathrm{mmol})$ in glacial acetic acid $(10 \mathrm{~mL})$ was stirred at $50-55^{\circ} \mathrm{C}$ for $3 \mathrm{~h}$. The reaction mixture was cooled. The resulting solid product was collected by filtration and recrystallized to obtain 20 (0.31 g, 70\%).

2.26. General Procedures for the Preparation of 11-substituted-4-methyl-2-phenyl-pyrimido $\left[3^{\prime}, 2^{\prime}: 4,5\right]$ thieno[3,2:

4,5]pyrimido[1, 6-b][1,2,4]triazepines (21a-21d). A mixture of compound (10) $(0.308 \mathrm{~g}, 1 \mathrm{mmol})$ and appropriate 3dimethyl-amino-1-(substituted)prop-2-enones 19a-19d $(1 \mathrm{mmol})$ in glacial acetic acid $(10 \mathrm{~mL})$ was refluxed for $10 \mathrm{~h}$. The reaction mixture was cooled and poured into ice-water; the precipitate was filtered and recrystallized from $\mathrm{DMF} /$ ethanol to obtain 21a-21d.

2.27. 2,11-Diphenyl-4-methyl-pyrimido $\left[3^{\prime}, 2^{\prime}: 4,5\right]$ thieno $[3,2$ : 4,5]pyrimido[1,6-b][1,2,4]triazepine (21a): Pale Reddish Brown Crystals. Yield 0.29 g (70\%), mp 194 C; IR: $v 1611$ $(\mathrm{C}=\mathrm{N}) \mathrm{cm}^{-1} ;{ }^{1} \mathrm{H}$ NMR $\left(\mathrm{CF}_{3} \mathrm{COOD}\right): \delta 2.22\left(3 \mathrm{H}, \mathrm{s}, \mathrm{CH}_{3}\right)$, $7.08(2 \mathrm{H}, \mathrm{d}, J=1.00 \mathrm{~Hz}, 10-\mathrm{H}), 7.48(2 \mathrm{H}, \mathrm{d}, J=1.00 \mathrm{~Hz}$, 9-H), 8.03-7.68, 7.59-7.53 (10H, m, phenyl-H), $9.51(1 \mathrm{H}, \mathrm{s}$, 6-H); MS: 420( $\left.\mathrm{M}^{+}, 100\right), 387(12), 366(48), 318(23), 308(30)$, 293(45), 278(29), 263(8), 210(14), 190(19), 120(9), 105(38), 77(56). Anal. Calcd. for $\mathrm{C}_{24} \mathrm{H}_{16} \mathrm{~N}_{6} \mathrm{~S}$ : C, 68.57; H, 3.80; N, 20.00. Found: C, $68.69 ; \mathrm{H}, 3.95 ; \mathrm{N}, 20.25 \%$.

2.28. 11-(2-Furyl)-4-methyl-2-phenyl-pyrimido $\left[3^{\prime}, 2^{\prime}: 4,5\right]$ thieno[3,2:4,5]pyrimido [1,6-b][1,2,4] triazepine (21b): Greenish Yellow Crystals. Yield 0.26g, (64\%) mp $244^{\circ} \mathrm{C}$; IR: $v 1619$ $(\mathrm{C}=\mathrm{N}) \mathrm{cm}^{-1} ;{ }^{1} \mathrm{H} \mathrm{NMR}\left(\mathrm{CF}_{3} \mathrm{COOD}\right): \delta 2.23\left(3 \mathrm{H}, \mathrm{s}, \mathrm{CH}_{3}\right)$,
$7.05(2 \mathrm{H}, \mathrm{d}, J=1.00 \mathrm{~Hz}, 10-\mathrm{H}), 6.60(1 \mathrm{H}, \mathrm{dd}, J=1.00$, $1.00 \mathrm{~Hz}, 4-\mathrm{H}$ of furyl $), 6.69(1 \mathrm{H}, \mathrm{d}, J=1.00 \mathrm{~Hz}, 3-\mathrm{H}$ of furyl), 7.91-7.81, 7.76-7.61 $(6 \mathrm{H}, \mathrm{m}, 9-\mathrm{H}$, and phenyl-H), 8.34 $(1 \mathrm{H}, \mathrm{d}, J=1.00 \mathrm{~Hz}, 5-\mathrm{H}$ of furyl), $9.53(1 \mathrm{H}, \mathrm{s}, 6-\mathrm{H}) ; \mathrm{MS}$ : 410 $\left(\mathrm{M}^{+}, 100\right), 356(42), 319(82), 293(48), 278(42), 250(18)$, 229(10), 205(13), 190(23), 174(18), 163(14), 129(25), 104(44), 95(51), 77(42). Anal. Calcd. for $\mathrm{C}_{22} \mathrm{H}_{14} \mathrm{~N}_{6} \mathrm{OS}$ : C, $64.39 ; \mathrm{H}, 3.41 ; \mathrm{N}, 20.48$. Found: C, 64.49; H, 3.68; N, 20.55\%.

2.29. 11-(2-Thienyl)-4-methyl-2-phenyl-pyrimido $\quad\left[3^{\prime}, 2^{\prime}: 4\right.$, 5]thieno[3,2:4,5]pyrimido[1,6-b][1,2,4]triazepine (21c): Yellowish Brown Crystals. Yield $0.27 \mathrm{~g}(63 \%), \mathrm{mp} 231^{\circ} \mathrm{C}$; IR: $v 1623(\mathrm{C}=\mathrm{N}) \mathrm{cm}^{-1} ;{ }^{1} \mathrm{H}$ NMR $\left(\mathrm{CF}_{3} \mathrm{COOD}\right): \delta 2.24(3 \mathrm{H}$, s, $\left.\mathrm{CH}_{3}\right), 7.33(2 \mathrm{H}, \mathrm{d}, J=1.00 \mathrm{~Hz}, 10-\mathrm{H}), 7.84(2 \mathrm{H}, \mathrm{d}, J=$ $1.00 \mathrm{~Hz}, 9-\mathrm{H}), 7.20(1 \mathrm{H}, \mathrm{dd}, J=1.00,1.00 \mathrm{~Hz}, 4-\mathrm{H}$ of thienyl), $7.13(1 \mathrm{H}, \mathrm{d}, J=1.00 \mathrm{~Hz}, 3-\mathrm{H}$ of thienyl), 7.91-7.90, 7.31-7.29 $(5 \mathrm{H}, \mathrm{m}$, phenyl-H), $8.11(1 \mathrm{H}, \mathrm{d}, J=1.0 \mathrm{~Hz}, 5-\mathrm{H}$ of thienyl), 9.54 (1H, s, 6-H); MS: 426( $\left.\mathrm{M}^{+}, 10\right), 399(5), 333(7), 319(100)$, 279(13), 213(2), 190(7), 121(3), 111(77), 77(8). Anal. Calcd. for $\mathrm{C}_{22} \mathrm{H}_{14} \mathrm{~N}_{6} \mathrm{~S}_{2}$ : C, 61.97; H, 3.28; N, 19.71. Found: C, 62.12; $\mathrm{H}, 3.38$; N, $19.87 \%$.

2.30. 11-(2-Pyrazinyl)-4-methyl-2-phenyl-pyrimido $\left[3^{\prime}, 2^{\prime}: 4,5\right]$ thieno[3,2:4,5]pyrimido[1,6-b][1,2,4] triazepine (21d): Brown Crystals. Yield $0.22 \mathrm{~g}(53 \%), \mathrm{mp} 240^{\circ} \mathrm{C}$; IR: $v 1624(\mathrm{C}=\mathrm{N})$ $\mathrm{cm}^{-1} ;{ }^{1} \mathrm{H}$ NMR $\left(\mathrm{CF}_{3} \mathrm{COOD}\right): \delta 2.32\left(3 \mathrm{H}, \mathrm{s}, \mathrm{CH}_{3}\right), 6.71$ $(2 \mathrm{H}, \mathrm{d}, J=1.00 \mathrm{~Hz}, 10-\mathrm{H}), 7.45(2 \mathrm{H}, \mathrm{d}, J=1.00 \mathrm{~Hz}, 9-$ $\mathrm{H}), 8.24(1 \mathrm{H}, \mathrm{d}, J=1.00 \mathrm{~Hz}, 6-\mathrm{H}$ of pyrazinyl), $8.41-8.36$, 7.93-7.77 (5H, m, phenyl-H), $8.49(1 \mathrm{H}, \mathrm{d}, J=1.00 \mathrm{~Hz}, 5-\mathrm{H}$ of pyrazinyl), $8.98(1 \mathrm{H}, \mathrm{s}, 3-\mathrm{H}$ of pyrazinyl), $9.19(1 \mathrm{H}, \mathrm{s}, 6-\mathrm{H})$; MS: 422( $\left.\mathrm{M}^{+}, 24\right), 395(9), 319(35), 293(100), 238(4), 215(10)$, 190(41), 163(22), 147(8), 104(19), 77(23). Anal. Calcd. for $\mathrm{C}_{22} \mathrm{H}_{14} \mathrm{~N}_{8} \mathrm{~S}: \mathrm{C}, 62.55 ; \mathrm{H}, 3.31 ; \mathrm{N}, 26.54$. Found: C, 62.74; $\mathrm{H}$, $3.48 ; \mathrm{N}, 26.78 \%$.

2.31. 11-(4-Pyridyl)-4-methyl-2-phenyl-pyrimido[3', $\left.2^{\prime}: 4,5\right]$ thieno[3,2:4,5]pyrimido[1,6-b][1,2,4] triazepine (21e). Method A: this compound was synthesized from compound (10) $(0.308 \mathrm{~g}, 1 \mathrm{mmol})$ in glacial acetic acid in a similar way to that described for the preparation of $21 \mathbf{a}-21 d$. It was recrystallized from DMF/ethanol to give pale brown crystals. Yield $0.29 \mathrm{~g},(68 \%) \mathrm{mp} 237^{\circ} \mathrm{C}$; IR: $v 1622(\mathrm{C}=\mathrm{N}) \mathrm{cm}^{-1}$; ${ }^{1} \mathrm{H}$ NMR $\left(\mathrm{CF}_{3} \mathrm{COOD}\right): \delta 3.19\left(3 \mathrm{H}, \mathrm{s}, \mathrm{CH}_{3}\right), 7.30(2 \mathrm{H}, \mathrm{d}, J=$ $1.0 \mathrm{~Hz}, 10-\mathrm{H}), 7.97(2 \mathrm{H}, \mathrm{d}, J=1.00 \mathrm{~Hz}, 3,5-\mathrm{H}$ of pyridyl), $8.14-8.12,7.50-7.38(5 \mathrm{H}, \mathrm{m}$, phenyl-H), $8.16(2 \mathrm{H}, \mathrm{d}, J=$ $1.00 \mathrm{~Hz}, 9-\mathrm{H}), 8.31(1 \mathrm{H}, \mathrm{s}, 6-\mathrm{H}), 8.54(2 \mathrm{H}, \mathrm{d}, J=1.00 \mathrm{~Hz}$, 2,6-H of pyridyl); MS: 421( $\left.\mathrm{M}^{+}, 100\right), 367(4), 333(8), 318(19)$, 308(44), 293(66), 278(42), 214(8), 198(10), 190(22), 163(12), 120(5), 104(22), 77(26). Anal. Calcd. for $\mathrm{C}_{23} \mathrm{H}_{15} \mathrm{~N}_{7} \mathrm{~S}$ : C, $65.55 ; \mathrm{H}, 3.56 ; \mathrm{N}, 23.27$. Found: C, 65.74; H, 3.68; N, 23.48\%. Method B: the filtrate from the above reaction 20 was poured into cold water $(20 \mathrm{~mL})$ and stirred for $15 \mathrm{~min}$. The resulting precipitate was collected by filtration and recrystallized to give $21 \mathrm{e}(0.026 \mathrm{~g}, 6.3 \%)$. Method C: a mixture of $20(0.439 \mathrm{~g}$, $1 \mathrm{mmol})$ and glacial acetic acid $(10 \mathrm{~mL})$ was refluxed for $10 \mathrm{~h}$. The reaction mixture was cooled and poured into ice-water; the precipitate was filtered and recrystallized to obtain $\mathbf{2 1 e}$ (0.202 g, 48\%). 
<smiles>Cc1nc(-c2ccccc2)[nH]c(=S)c1C#N</smiles>

1 $+\quad \mathrm{ClCH}_{2} \mathrm{CN} \underset{\text { Room temperature }}{\stackrel{\mathrm{DMF} / \mathrm{K}_{2} \mathrm{CO}_{3}}{\longrightarrow}}$ $4 \mathrm{~h}$

2<smiles>Cc1nc(-c2ccccc2)nc(SCCC#N)c1C#N</smiles>

$\downarrow$<smiles>Cc1nc(-c2ccccc2)nc2sc(C#N)c(N=C[OH2+])c12</smiles>

4

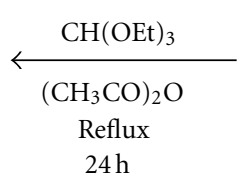

$24 \mathrm{~h}$

SCHEME 1

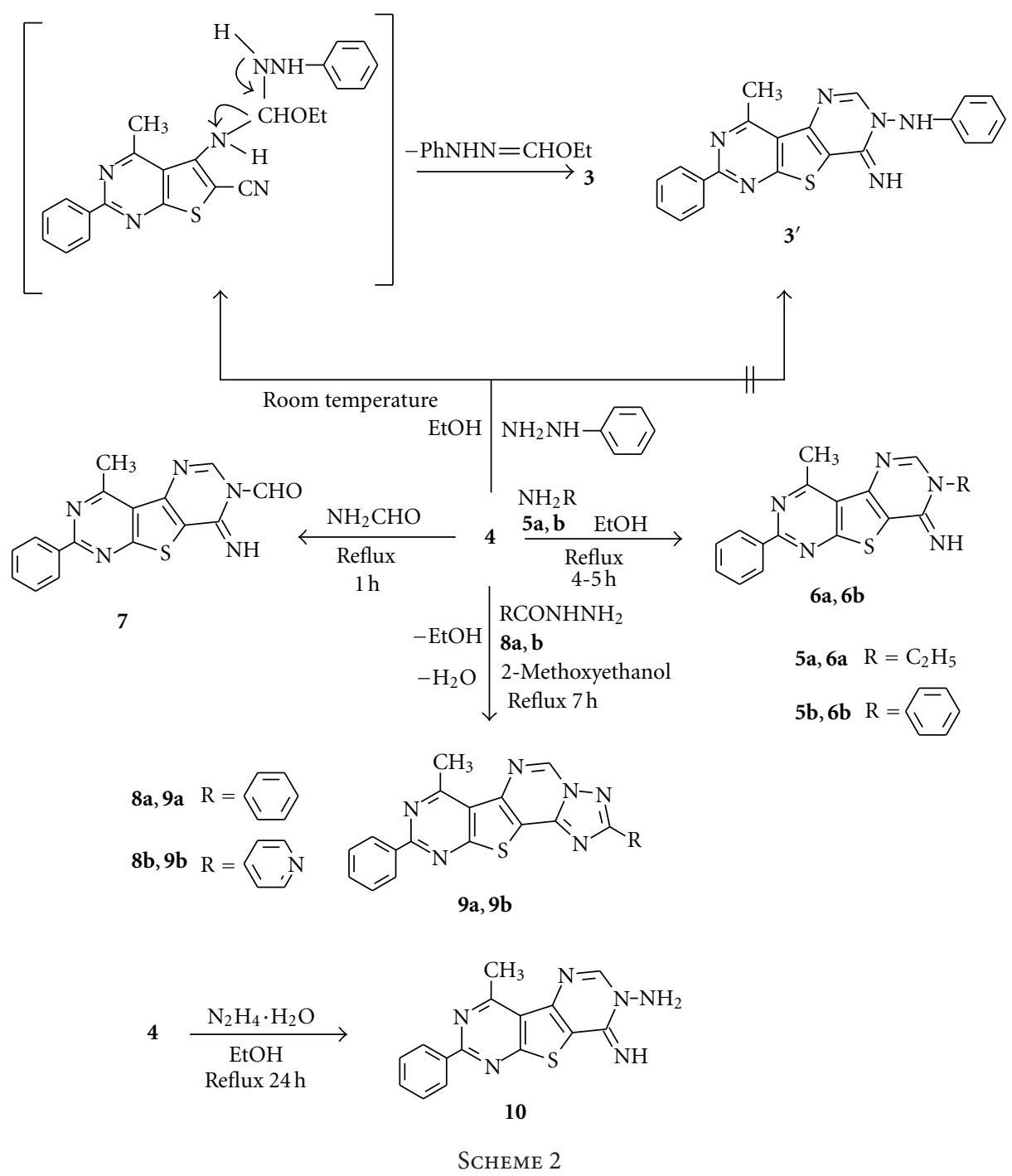




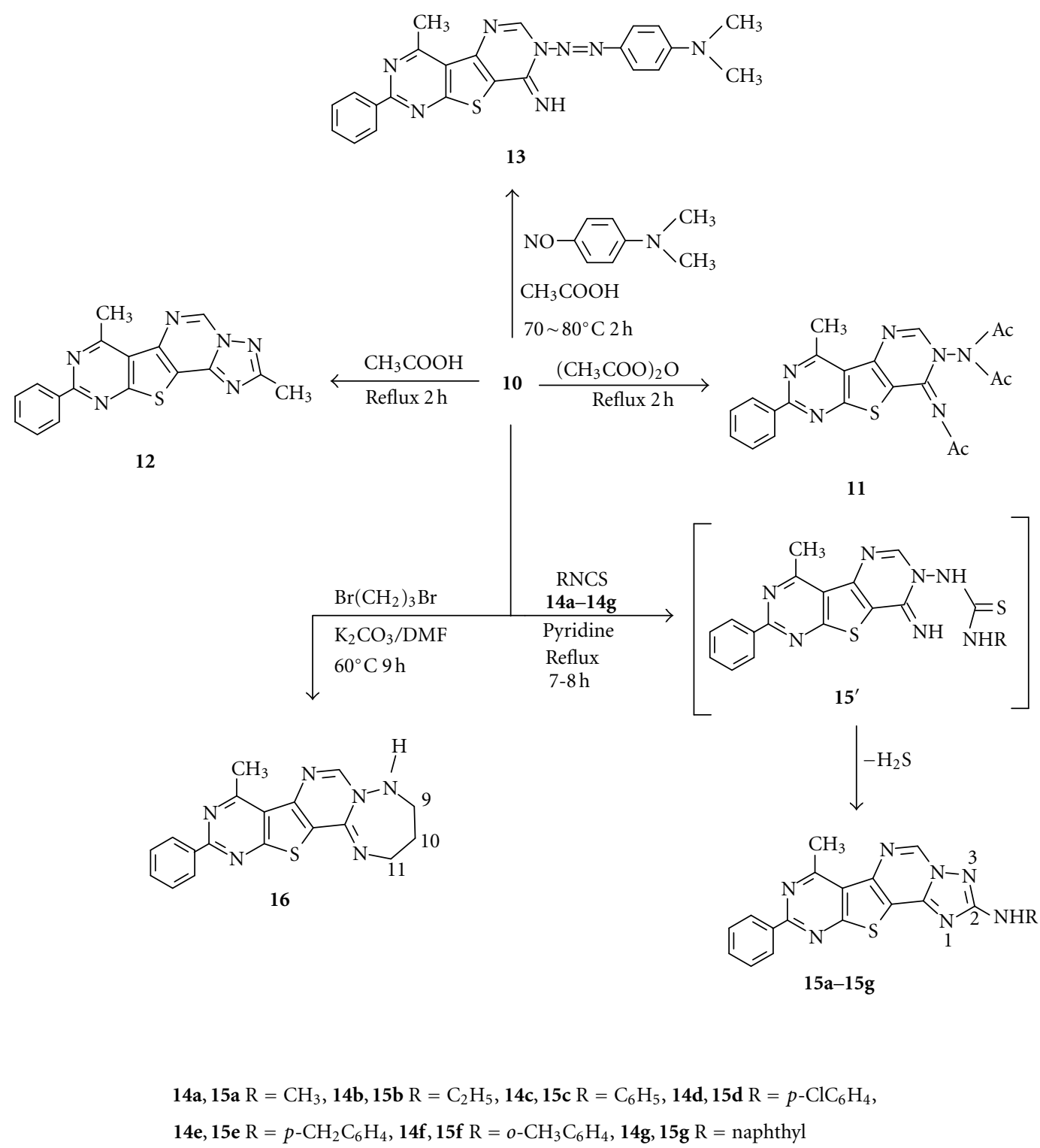

SCHEME 3

\section{Results and Discussion}

Cyclization of thioxopyrimidine $\mathbf{1}$ with chloroacetonitrile 2 in DMF in the presence of excess anhydrous potassium carbonate formed the nonisolable S-alkylated intermediate, which via nucleophilic substitution and intramolecular cyclocondensation afforded the 5-amino-6-cyanothieno[2,3d]pyrimidine 3 , and the latter reacted with triethyl orthoformate to give the 5-ethoxymethyleneamino-thieno[2,3d]pyrimidines 4 (Scheme 1). Moreover, the reactivity of compound 4 towards amino compounds was also investigated. In treatment of $\mathbf{4}$ with phenylhydrazine in ethanol, an addition product formed, from which elimination of ethyl formate phenylhydrazone gave the compound 3 instead of the compound $\mathbf{3}^{\prime}$, while with amino compounds $\mathbf{5 a}, \mathbf{5} \mathbf{b}$, and formamide afforded the corresponding 7-substituted8-imino-pyrimido[3,2: 4,5] thieno[2,3-d]pyrimidines $\mathbf{6 a}, \mathbf{6} \mathbf{b}$, and 7 , respectively (Scheme 2). The structure of compounds $\mathbf{6 a}, \mathbf{6 b}$, and 7 was established on the basis of their elemental analysis and spectra data. The IR spectra of compounds 6a, 6b, and 7 showed the characteristic absorption band at $3310-3301 \mathrm{~cm}^{-1}$ for the $\mathrm{NH}$ group. In addition, the ${ }^{1} \mathrm{H}$ NMR spectra $\left(\mathrm{CDCl}_{3}\right)$ of compound $\mathbf{6 a}$ revealed a triplet at $\delta 1.47(3 \mathrm{H}, \mathrm{s})$ and a quartet at $4.10(2 \mathrm{H}, \mathrm{q})$, which were readily assigned to the ethyl group $\left(\mathrm{CH}_{2} \mathrm{CH}_{3}\right)$ and a singlet at $7.90(1 \mathrm{H}, \mathrm{s})$ which was assigned to the hydrogen attached at $\mathrm{C}_{6}$ of the pyrimidine ring, which was also confirmed by the mass spectrum $\mathrm{m} / \mathrm{z} 321\left(\mathrm{M}^{+}\right)$. Further, the ${ }^{1} \mathrm{H}$ NMR spectra (DMSO- $\mathrm{d}_{6}$ ) of compound 7 revealed three singlets at $\delta 8.33(1 \mathrm{H}, \mathrm{s}), 8.54(1 \mathrm{H}, \mathrm{s})$, and $8.86(1 \mathrm{H}, \mathrm{s})$, which 


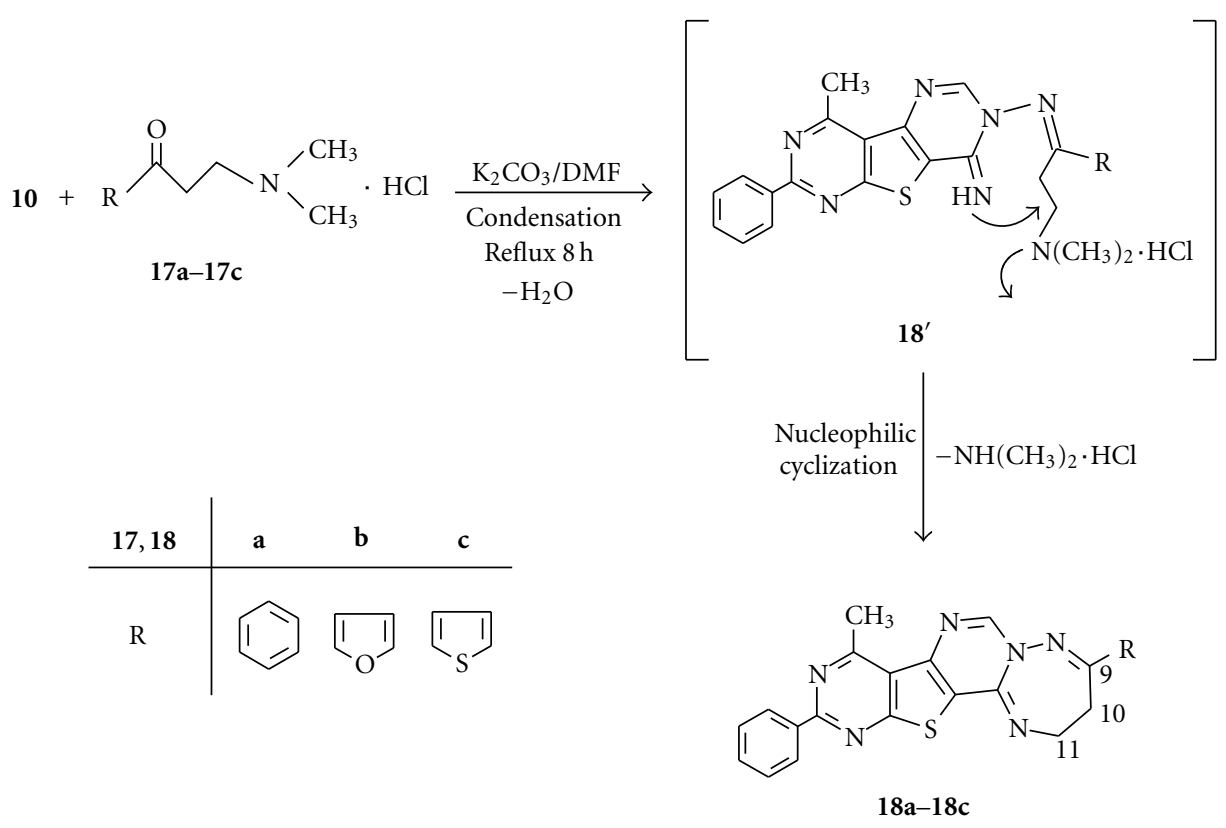

SCHeme 4

were readily assigned to the $\mathrm{NH}, \mathrm{HCO}$, and $\mathrm{C}_{6}-\mathrm{H}$ protons, respectively. Nevertheless, the compound $\mathbf{4}$ was cyclized with acid hydrazides $\mathbf{8 a}, \mathbf{8 b}$ under different conditions to form a new tetracyclic compound. Thus, compound $\mathbf{4}$ with acid hydrazides $\mathbf{8 a}, \mathbf{8 b}$ in refluxing 2-methoxyethanol afforded the corresponding 2-substituted-pyrimido $\left[3^{\prime}, 2^{\prime}: 4,5\right]$ thieno[3,2e] $[1,2,4]$ triazolo[1,5-c]pyrimidines $9 \mathbf{a}, \mathbf{9 b}$. The ${ }^{1} \mathrm{H}$ NMR spectra $\left(\mathrm{CF}_{3} \mathrm{COOD}\right)$ of the compounds $\mathbf{9 a}, \mathbf{9 b}$, which showed a singlet at $\delta 9.93(1 \mathrm{H}, \mathrm{s})$ and $9.83(6 \mathrm{H}, \mathrm{s})$, were readily assigned to the hydrogen attached at $\mathrm{C}_{6}$ of the pyrimidine ring, respectively. Hydrazinolysis of compound $\mathbf{4}$ in ethanol yielded the key intermediate (10) for the preparation of new triazoles and triazepines (Scheme 2). Moreover, the IR spectra of compound (10) showed the characteristic absorption band at 3315 and $3247 \mathrm{~cm}^{-1}$ for the $\mathrm{NH}_{2}$ and $\mathrm{NH}$ groups, respectively. The ${ }^{1} \mathrm{H}$ NMR spectra $\left(\mathrm{CF}_{3} \mathrm{COOD}\right)$ of compound (10) revealed three singlets at $\delta 5.12(1 \mathrm{H}, \mathrm{s}), 9.23$ $(1 \mathrm{H}, \mathrm{s})$, and $8.69(1 \mathrm{H}, \mathrm{s})$, which were readily assigned to the $\mathrm{NH}_{2}, \mathrm{NH}$, and $\mathrm{C}_{6}-\mathrm{H}$ protons, respectively, which was also confirmed by the mass spectrum $m / z 308\left(\mathrm{M}^{+}\right)$.

Next, as described in Scheme 3, several pyrimido $[3,2: 4,5]$ thieno[2,3-d]pyrimidines (PTP) substituted at positions 7 and 8 with different heterocyclic residues were obtained via treatment of compound (10) with different reagents. Thus, upon heating compound (10) in refluxing acetic anhydride, introduced three acetyl groups afforded the 8-(acetylamino)-7-diacetylamino-4-methyl-2-phenylpyrimido $[3,2: 4,5]$ thieno[2,3-d]pyrimidine (11). Also, the compound (10) cyclized in glacial acetic acid at $70-80^{\circ} \mathrm{C}$ for $2 \mathrm{~h}$ afforded the 2,7-dimethyl-pyrimido[ $\left.3^{\prime}, 2^{\prime}: 4,5\right]$ thieno[3,2e] $[1,2,4]$ triazolo[1,5-c]pyrimidine (12). The structure of compounds (11),(12) was established on the basis of their elemental analysis and spectral data. The IR spectra of compounds (11),(12) indicated the complete disappearance of $\mathrm{NH}$ and $\mathrm{NH}_{2}$ groups. The ${ }^{1} \mathrm{H}$ NMR spectra $\left(\mathrm{CF}_{3} \mathrm{COOD}\right)$ of the compound (11), which showed additional two signals at $\delta 2.32(3 \mathrm{H}, \mathrm{s})$ and $2.56(6 \mathrm{H}, \mathrm{s})$, which were assigned to the protons $\mathrm{COCH}_{3}$ attached at imino and amino groups of PTP moiety, respectively, were also confirmed by the mass spectrum $\mathrm{m} / z 434\left(\mathrm{M}^{+}\right)$. Moreover, reaction of (10) with $\mathrm{N}, \mathrm{N}$ - dimethyl-4-nitrosoaniline in refluxing glacial acetic acid afforded the 7-(4-dimethyl-aminophenyl)azo-8-imino4-methyl-2-phenyl-7,8-dihydropyrimido[3,2:4,5] thieno[2,3$d$ ]pyrimidine 13, because the molecular ion $\mathrm{m} / z 440$ of compound 13 is unstable and could not be recorded in the electron impact mass spectra but showed the presence of the ion peaks $m / z 412, m / z 397, m / z 319$, and $m / z 293$. The possible mass fragmentation pathway of compound $\mathbf{1 3}$ is shown in Scheme 6. In addition, the structure of compound 13 was supported by the ${ }^{1} \mathrm{H}$ NMR spectra, which showed a sharp singlet at $\delta 3.51(6 \mathrm{H}, \mathrm{s})$ assigned to the $\mathrm{N}\left(\mathrm{CH}_{3}\right)_{2}$ protons.

On the other hand, the 2-(substituted-amino)pyrimido $\left[3^{\prime}, 2^{\prime}: 4,5\right]$ thieno $[3,2-e][1,2,4]$ triazolo[ $[1,5-c]$ pyr-

imidines $15 \mathbf{a}-\mathbf{1 5} \mathrm{g}$ were obtained by intramolecular cyclization of compound (10) with appropriate isothiocyanates $\mathbf{1 4 a - 1 4} \mathbf{g}$ in refluxing pyridine (Scheme 3). Obviously this reaction proceeded via the thiourea intermediate $15^{\prime}$ with concomitant dehydrosulfurization. The structure of compounds $15 \mathbf{a}-\mathbf{1 5} \mathrm{g}$ was established on the basis of their elemental analysis and spectral data. The ${ }^{1} \mathrm{H}$ NMR spectra of $\mathbf{1 5 a}-\mathbf{1 5 g}$ showed a singlet at $\delta$ 9.98-8.79 $(1 \mathrm{H}, \mathrm{s})$ assigned to the hydrogen attached at $\mathrm{C}_{5}$ of the pyrimidotriazole ring. In addition, the ${ }^{1} \mathrm{H}$ NMR spectra of compound $15 \mathbf{b}$ revealed a triplet at $\delta 1.92$ $(3 \mathrm{H}, \mathrm{s})$ and a quartet at $4.92(2 \mathrm{H}, \mathrm{q})$, which were readily assigned to the ethyl group $\left(\mathrm{CH}_{2} \mathrm{CH}_{3}\right)$ and a singlet at $9.15(1 \mathrm{H}, \mathrm{br})$ which was assigned to the $\mathrm{NH}$ group. Also, 

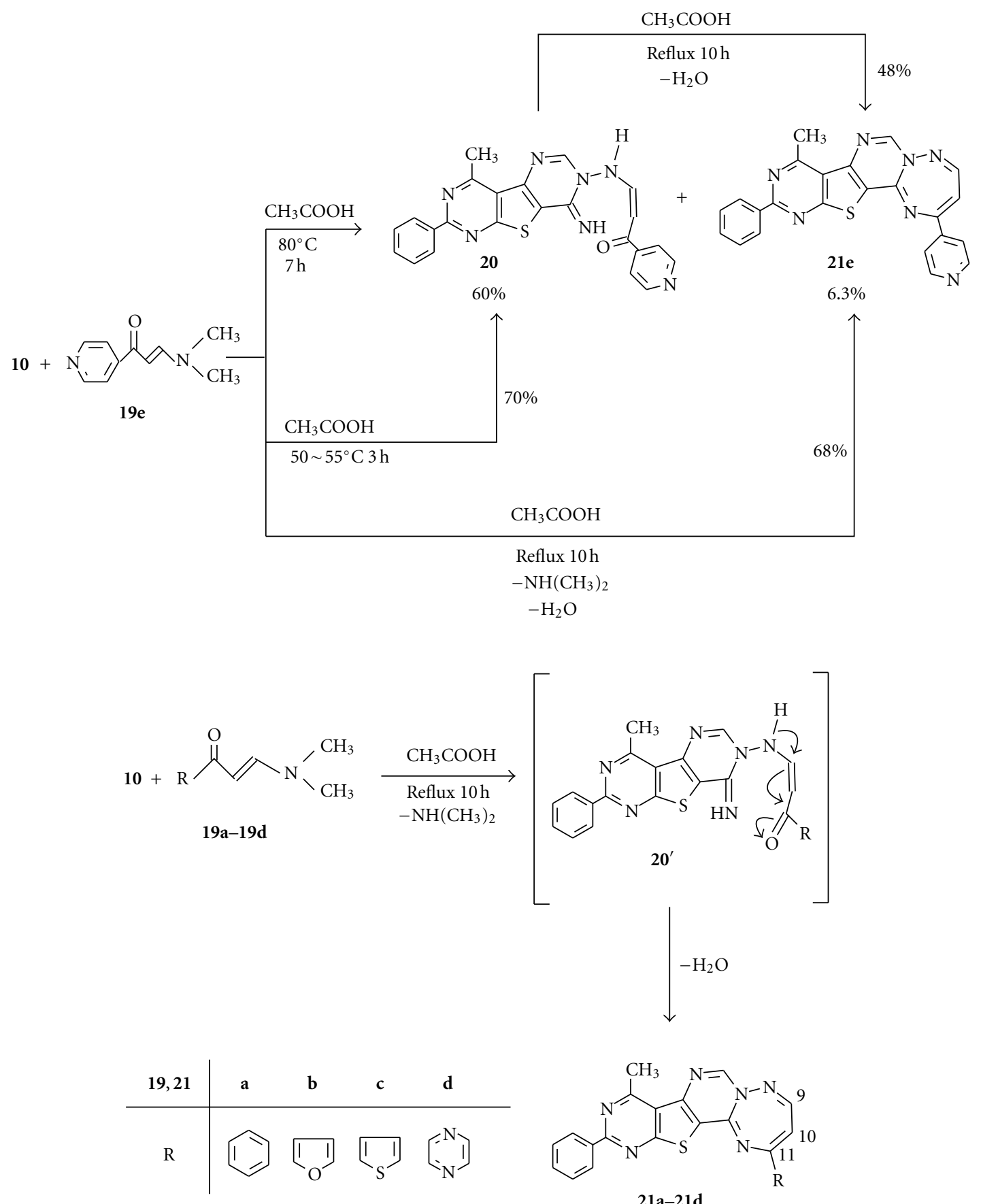

SCHEME 5

it has been observed that electron impact (EI) spectral has many common features. Compounds $15 \mathrm{a}-\mathbf{1 5 g}$ exhibited $\mathrm{m} / \mathrm{z} 332, \mathrm{~m} / \mathrm{z} 304, \mathrm{~m} / \mathrm{z} 293, \mathrm{~m} / \mathrm{z} 278$, and $\mathrm{m} / \mathrm{z} 174$ piece peaks. Next, cyclocondensation of compound (10) with 1,3-dibromopropane in DMF in the presence of excess anhydrous potassium carbonate at $60^{\circ} \mathrm{C}$ form the $9,10,11$ trihydropyrimido $\left[3^{\prime}, 2^{\prime}: 4,5\right]$ thieno[3,2:4,5] pyrimido[1,6-

b] $[1,2,4]$ triazepine 16. In particular, the ${ }^{1} \mathrm{H}$ NMR spectra of compound $\mathbf{1 6}$ revealed two additional triplets at $\delta 3.42$ $(2 \mathrm{H}, \mathrm{t})$ and $4.42(2 \mathrm{H}, \mathrm{t})$, which were readily assigned to the hydrogen attached at $\mathrm{C}_{11}$ and $\mathrm{C}_{9}$ of the triazepine ring, respectively, a singlet at $\delta 10.12(1 \mathrm{H}, \mathrm{br})$ assigned to the $\mathrm{NH}$ group, and a multiplet at $\delta 2.32(2 \mathrm{H}, \mathrm{m})$ assigned to the hydrogen attached at $\mathrm{C}_{10}$ of the triazepine ring, which was also confirmed by the mass spectrum $\mathrm{m} / z 348\left(\mathrm{M}^{+}\right)$.

Work was further extended to study the behavior of compound (10) towards the different reagents with a view to synthesizing various heterocyclic ringsystems. Thus, treatment of compound (10) with Mannich bases $[30,31] \mathbf{1 7} \mathbf{a}-\mathbf{1 7} \mathbf{c}$ in DMF in the presence 

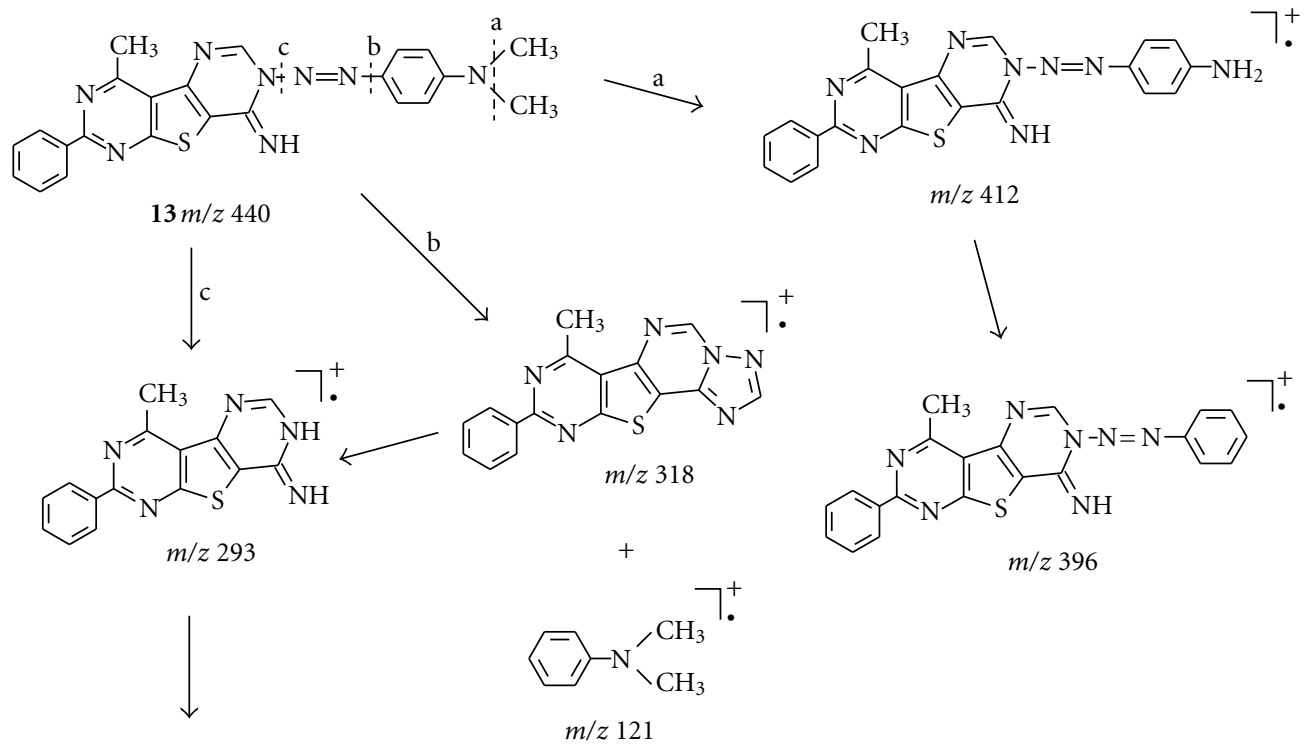<smiles>Cc1nc(-c2ccccc2)nc2sc3cncnc3c12</smiles>

SCHEME 6

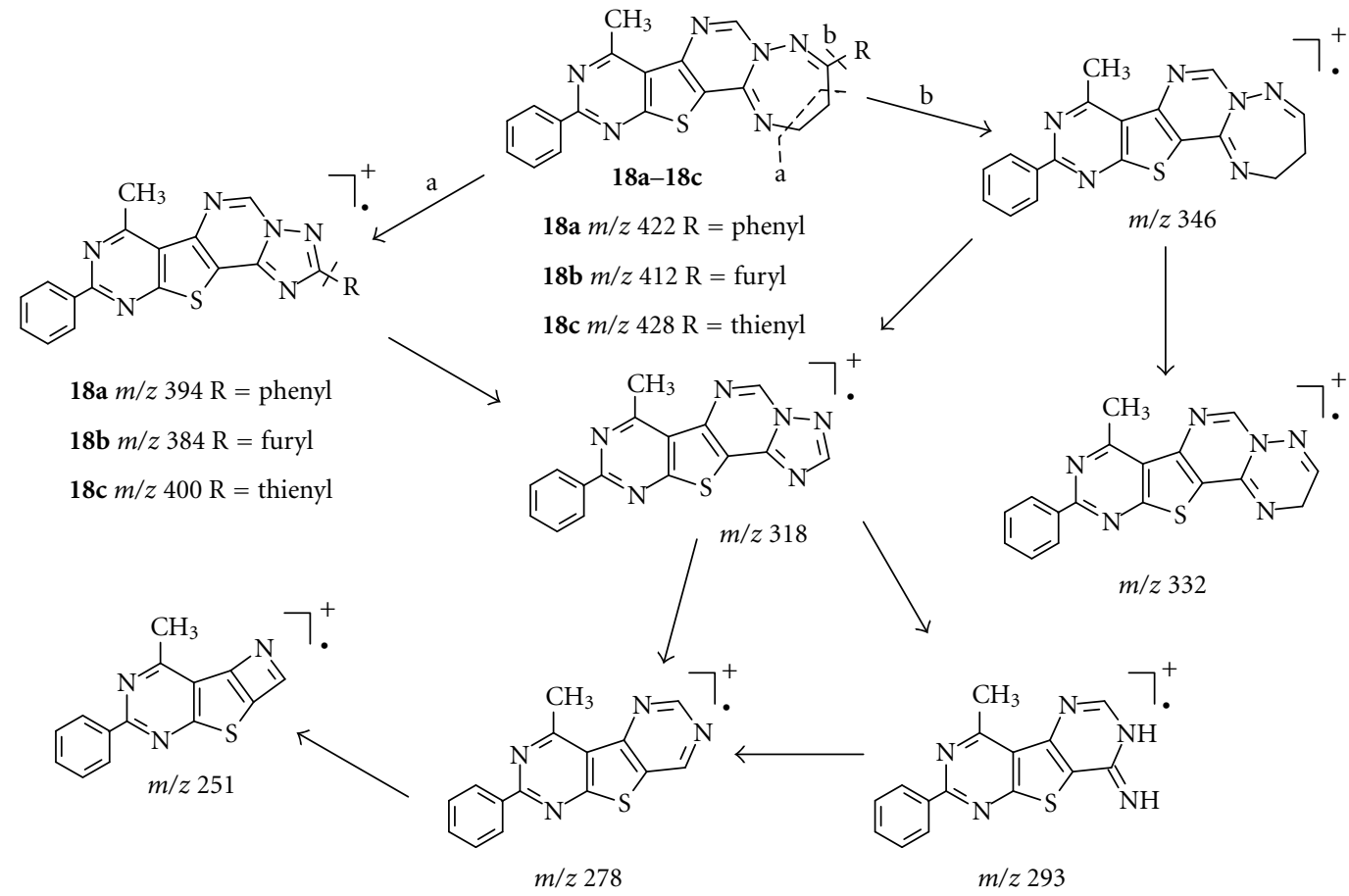

SCHEMe 7

of excess anhydrous potassium carbonate afforded the corresponding 9-substituted-10,11-dihydropyrimido thieno[3,2:4,5]pyrimido[ $1,6-b][1,2,4]$ triazepines $\mathbf{1 8 a}-\mathbf{1 8 c}$ (Scheme 4). The mechanism involves the condensation of amino group in compound (10) with the carbonyl group, followed by dehydration and subsequent nucleophilic cyclization with loss of $\mathrm{N}, \mathrm{N}$-dimethylamine hydrochloride [26]. The ${ }^{1} \mathrm{H}$ NMR spectra of compounds $\mathbf{1 8 a}-\mathbf{1 8 c}$ revealed two additional triplets at $\delta 3.22-3.28(2 \mathrm{H}, \mathrm{t})$ and $4.19-4.23$ $(2 \mathrm{H}, \mathrm{t})$, which were readily assigned to the hydrogen attached 
at $\mathrm{C}_{10}$ and $\mathrm{C}_{11}$ of the triazepine ring, respectively. These structures get further support from mass spectroscopy. It has been observed that Electron Impact (EI) spectral has many common features. Compounds 18a-18c exhibited $\mathrm{m} / z$ 345, $\mathrm{m} / z$ 332, $\mathrm{m} / z$ 318, $\mathrm{m} / z 293, \mathrm{~m} / z 278, \mathrm{~m} / z 250, \mathrm{~m} / z$ $215, m / z 190$, and $m / z 163$ piece peaks. The possible mass fragmentation pathway of compounds $\mathbf{1 8 a - 1 8 c}$ is shown in Scheme 7.

Furthermore, the behavior of compound (10) with enaminone derivatives 19a-19e was also investigated (Scheme 5). To optimize the reaction temperature, the reaction of compound (10) and enaminone 19e was studied in glacial acetic acid, at different temperatures such as $50^{\circ} \mathrm{C}-55^{\circ} \mathrm{C}$, $80^{\circ} \mathrm{C}$, and reflux, respectively. It has been found that the treatment of compound (10) and enaminone $19 \mathrm{e}$ carried out at $50-55^{\circ} \mathrm{C}$ for $3 \mathrm{~h}$ only afforded the open-chain product 20 in $70 \%$ yield, while at $80^{\circ} \mathrm{C}$ for $7 \mathrm{~h}$ incomplete cyclocondensation was observed (as examined by TLC (ethyl acetate and hexane $(3: 7)))$; work-up of the reaction mixture yielded a mixture of two products 20 (60\% yield) and 21 e (6.3\% yield) which were separated. Also, it was interesting to find that prolonging the reaction time $(10 \mathrm{~h})$ and increasing the reaction temperature (reflux), the yield for compound 21 e increased greatly from $6.3 \%$ to $68 \%$.

The structures of $\mathbf{2 0}$ and 21e were established on the basis of microanalysis and spectra data as well as comparison (IR, mixed mp, TLC (ethyl acetate and hexane (3:7)). The IR spectra of the compound $\mathbf{2 0}$ showed the characteristic absorption band at $3215 \mathrm{~cm}^{-1}$ for the $\mathrm{NH}$ group and at $1668 \mathrm{~cm}^{-1}$ for the $\mathrm{C}=\mathrm{O}$ group. The ${ }^{1} \mathrm{H}$ NMR spectra of compound 20 showed a doublet at $\delta 4.00(1 \mathrm{H}, \mathrm{d})$ assigned to the $\mathrm{COCH}=$ of 1 -(4-pyridinyl)prop-2-enone moiety and a multiplet at $\delta 8.78-8.74,8.26-8.07(6 \mathrm{H}, \mathrm{m})$ assigned to the $\mathrm{NCH}=$ of 1-(4-pyridinyl)prop-2-enone moiety and phenyl protons. In addition, the IR spectra of compound 21e indicated the absence of the $\mathrm{NH}_{2}$ and $\mathrm{NH}$ groups. The ${ }^{1} \mathrm{H}$ NMR spectra of compound 21e revealed two doublets at $\delta$ $7.52(1 \mathrm{H}, \mathrm{d})$ and $8.16(1 \mathrm{H}, \mathrm{d})$, which were readily assigned to the hydrogen attached at $\mathrm{C}_{10}$ and $\mathrm{C}_{9}$ of the triazepine ring, respectively. The structure of compound $21 \mathrm{e}$ was further confirmed via an independent synthesis of compound $21 \mathbf{e}$ by reaction of compound $\mathbf{2 0}$ in glacial acetic acid under reflux $10 \mathrm{~h}$ to afford a product identical in all respects ( $\mathrm{mp}$, mixed mp, TLC (ethyl acetate and hexane (3:7)) and spectra), with those of compounds 21 e in $48 \%$ yield. Moreover, treatment of compound (10) with enaminones 19a-19d in glacial acetic acid under reflux afforded the corresponding 11substituted-pyrimido $\left[3^{\prime}, 2^{\prime}: 4,5\right]$ thieno[3,2:4,5]pyrimido[1,6b] $[1,2,4]$ triazepines 21a-21d, respectively (Scheme 5). The formation of compound 21a-e would involve an initial Michael addition of the exocyclic amino group in compound (10) to the activated double bond in enaminone 19 to form the intermediate $\mathbf{2 0}$, which then undergoes cyclization and aromatization via loss of both water and N,N-dimethylamine [26] affording the final product 21a-21e. The ${ }^{1} \mathrm{H}$ NMR spectra of compounds $21 \mathbf{a}-21 \mathrm{e}$ revealed two additional doublets at $\delta 7.33-6.71(1 \mathrm{H}, \mathrm{d})$ and $8.16-7.45(1 \mathrm{H}, \mathrm{d})$, which were readily assigned to the hydrogen attached at $C_{10}$ and
$\mathrm{C}_{9}$ of the triazepine ring, respectively, and a singlet at $\delta$ 9.54-8.31 $(1 \mathrm{H}, \mathrm{s})$ assigned to the hydrogen attached at $\mathrm{C}_{6}$ of the triazepine ring. These structures get further support from mass spectroscopy.

\section{Conclusion}

In conclusion, 7-amino-8-imino-pyrimido[3,2:4,5] thieno pyrimidine (10) has been shown to be a useful building block for the synthesis of several novel 2(substituted-amino)-pyrimido $\left[3^{\prime}, 2^{\prime}: 4,5\right]$ thieno[3,2-e]

$[1,2,4]$ triazolo $[1,5-c]$ pyrimidines $\mathbf{1 5 a}-\mathbf{1 5 g}$, (tri)dihydropyrimido $\left[3^{\prime}, 2^{\prime}: 4,5\right]$ thieno[3,2:4,5]pyrimido $[1,6-b][1,2,4]$ triazepines $\mathbf{1 6}$ and $\mathbf{1 8 a}-\mathbf{1 8 c}$. On the other hand, the 11 -substituted-pyrimido $\left[3^{\prime}, 2^{\prime}: 4,5\right]$ thieno[3,2:4,5]pyrimido[1,6-b][1,2,4] triazepines 21a-21e were also obtained from compound (10).

\section{Acknowledgments}

The authors are grateful to the high-valued instrument of the Center of National Taiwan Normal University for measuring the data of spectroscopy. They also want to thank the National Science Council of Taiwan (NSC 97-2113-M-253-001) for their financial support.

\section{References}

[1] S. Bondock, W. Fadaly, and M. A. Metwally, "Enaminonitrile in heterocyclic synthesis: synthesis and antimicrobial evaluation of some new pyrazole, isoxazole and pyrimidine derivatives incorporating a benzothiazole moiety," European Journal of Medicinal Chemistry, vol. 44, no. 12, pp. 4813-4818, 2009.

[2] A. P. Keche, G. D. Hatnapure, R. H. Tale, A. H. Rodge, S. S. Birajdar, and V. M. Kamble, "A novel pyrimidine derivatives with aryl urea, thiourea and sulfonamide moieties: synthesis, anti-inflammatory and antimicrobial evaluation," Bioorganic \& Medicinal Chemistry Letters, vol. 22, no. 10, pp. 3445-3448, 2012.

[3] X. J. Song, Y. Shao, and X. G. Dong, "Microwave-assisted synthesis of some novel fluorinated pyrazolo[3,4-d] pyrimidine derivatives containing 1,3,4-thiadiazole as potential antitumor agents," Chinese Chemical Letters, vol. 22, no. 9, pp. 1036-1038, 2011.

[4] K. M. Amin, F. M. Awadalla, A. A. M. Eissa, S. M. AbouSeri, and G. S. Hassan, "Design, synthesis and vasorelaxant evaluation of novel coumarin-pyrimidine hybrids," Bioorganic \& Medicinal Chemistry, vol. 19, no. 20, pp. 6087-6097, 2011.

[5] E. P. da S. Falcão, S. J. de Melo, R. M. Srivastava, M. T. J. Catanho, and S. C. D. Nascimento, "Synthesis and antiinflammatory activity of 4-amino-2-aryl-5-cyano-6-\{3- and 4-(N-phthalimidophenyl)\} pyrimidines," European Journal of Medicinal Chemistry, vol. 41, no. 2, pp. 276-282, 2006.

[6] J. M. Cox, J. H. Marsden, R. A. Burrell, and N. S. Elmure, Chemical Abstracts, vol. 87, Article ID 128906, 1977, German Offen Patent 2, 654, 090, 1976.

[7] P. Schmidt and K. Eichenberger, Chemical Abstracts, vol. 75, Article ID 88638, 1971, German Offen Patent 2, 060, 968, 1970.

[8] J. B. Press and R. K. Russel, Chemical Abstracts, vol. 107, Article ID 115604, 1987, US Patent 4, 670, 560, 1986. 
[9] B. V. Ashalatha, B. Narayana, K. K. Vijaya Raj, and N. Suchetha Kumari, "Synthesis of some new bioactive 3amino-2-mercapto-5,6,7,8-tetrahydro[1]benzothieno[2,3d]pyrimidin-4(3H)-one derivatives," European Journal of Medicinal Chemistry, vol. 42, no. 5, pp. 719-728, 2007.

[10] F. F. Janssens, L. E. J. Kennis, J. F. Hens, J. L. G. Torremans, and G. S. M. Diels, Chemical Abstracts, vol. 109, Article ID 37821, 1988, US Patent 4, 695, 575, 1987.

[11] M. Gupta, S. Paul, and R. Gupta, "Efficient and novel one-pot synthesis of antifungal active 1-substituted-8-aryl-3-alkyl/aryl$4 H$-pyrazolo[4,5- $f][1,2,4]$ triazolo $[4,3-b][1,2,4]$ triazepines using solid support," European Journal of Medicinal Chemistry, vol. 46, no. 2, pp. 631-635, 2011.

[12] M. Gupta, "Efficient synthesis of antifungal active 9substituted-3-aryl-5H,13aH-quinolino[3,2-f][1,2,4]triazolo $[1,2,4]$ triazepines in ionic liquids," Bioorganic \& Medicinal Chemistry Letters, vol. 21, no. 16, pp. 4919-4923, 2011.

[13] D. M. Bailey, Chemical Abstracts, vol. 75, Article ID 140910, 1971, US 3, 607, 866, 1971.

[14] O. Bruno, C. Brullo, S. Schenone et al., "Progress in 5H[1] benzopyrano $[4,3-d]$ pyrimidin-5-amine series: 2-methoxy derivatives effective as antiplatelet agents with analgesic activity," Il Farmaco, vol. 57, no. 9, pp. 753-758, 2002.

[15] A. Padmaja, T. Payani, G. D. Reddy, and V. Padmavathi, "Synthesis, antimicrobial and antioxidant activities of substituted pyrazoles, isoxazoles, pyrimidine and thioxopyrimidine derivatives," European Journal of Medicinal Chemistry, vol. 44, no. 11, pp. 4557-4566, 2009.

[16] Q. Chen, X. L. Zhu, L. L. Jiang, Z. M. Liu, and G. F. Yang, "Synthesis, antifungal activity and CoMFA analysis of novel 1,2,4-triazolo[1,5-a]pyrimidine derivatives," European Journal of Medicinal Chemistry, vol. 43, no. 3, pp. 595-603, 2008.

[17] H. W. Lee, B. Y. Kim, J. B. Ahn et al., "Molecular design, synthesis, and hypoglycemic and hypolipidemic activities of novel pyrimidine derivatives having thiazolidinedione," European Journal of Medicinal Chemistry, vol. 40, no. 9, pp. 862-874, 2005.

[18] T. Mohamed and P. P. N. Rao, "Design, synthesis and evaluation of 2,4-disubstituted pyrimidines as cholinesterase inhibitors," Bioorganic and Medicinal Chemistry Letters, vol. 20, no. 12, pp. 3606-3609, 2010.

[19] D. Gala, D. J. DiBenedetto, M. Kugleman, and M. S. Puar, "Pyrimidine to guanine PDE inhibitors: determination of chemical course via structure elucidation," Tetrahedron Letters, vol. 44, no. 13, pp. 2717-2720, 2003.

[20] A. Mayasundari and N. Fujii, "Efficient formation of 4,6disubstituted pyrrolo[2,3- $d]$ pyrimidines: a novel route to TWS119, a glycogen synthase kinase- $3 \beta$ inhibitor," Tetrahedron Letters, vol. 51, no. 27, pp. 3597-3598, 2010.

[21] A. Angelucci, S. Schenone, G. L. Gravina et al., "Pyrazolo[3,4d]pyrimidines c-Src inhibitors reduce epidermal growth factorinduced migration in prostate cancer cells," European Journal of Cancer, vol. 42, no. 16, pp. 2838-2845, 2006.

[22] R. A. Mathes and F. D. Stewart, ChemicalAbstracts, vol. 45, Article ID 4273c, 1951, (B. F. Goodrich Co.) US Patent 2, 535, 858, 1950.

[23] W. Seebacher, G. Michl, and R. Weis, "Synthesis of new triazepinethiones," Tetrahedron Letters, vol. 43, no. 42, pp. 7481-7483, 2002.

[24] E. A. Savel'eva, Y. A. Rozin, M. I. Kodess et al., "Synthesis of mesoionic $[1,2,3]$ triazolo[5,1- $d][1,2,5]$ triazepines," Tetrahedron, vol. 60, no. 25, pp. 5367-5372, 2004.
[25] M. Gupta, S. Paul, and R. Gupta, "Efficient and novel one-pot synthesis of antifungal active 1-substituted-8-aryl-3-alkyl/aryl$4 H$-pyrazolo[4,5-f][1,2,4] triazolo[4,3-b][1,2,4] triazepines using solid support," European Journal of Medicinal Chemistry, vol. 46, no. 2, pp. 631-635, 2011.

[26] Y. W. Ho and C. T. Yao, "Synthesis of some new 6,8disubstituted 7,8-dihydro-pyrimido[2,3:4,3] pyrazolo[1,5-a] pyrimidines and 6,7,8-trisubstituted pyrimido[2,3:4,3] pyrazolo[1,5-a]pyrimidine derivatives," Journal of the Chinese Chemical Society, vol. 50, no. 2, pp. 283-296, 2003.

[27] Y. W. Ho, "5-(1-Pyrrolyl)-2-phenylthieno[2,3- $d]$ pyrimidine as building block in heterocyclic synthesis: novel synthesis of some pyrazoles, pyrimidines, imidazo[1,2-a]pyrimidines, pyrazolo[1,5-a]pyrimidines, pyrido-(pyrimido) pyrazolo[1,5a]pyrimidines, 1,2,4-triazolo[1,5-a]pyrimidine and a 1,2,3,4tetrazolo[1,5-a]pyrimidine derivative," Journal of the Chinese Chemical Society, vol. 54, no. 4, pp. 1075-1085, 2007.

[28] Y. W. Ho and M. C. Suen, "Synthesis and structure of novel thieno[2,3-d]pyrimidine derivatives containing 1,3,4oxadiazole moiety," Journal of the Chinese Chemical Society, vol. 56, no. 2, pp. 408-415, 2009.

[29] Y. W. Ho and W. H. Yao, "The synthesis and spectral characteristics of novel 6-(2-substituted-1,3,4-oxadiazol-5yl)-2-phenylthieno[2,3-d]pyrimidine fluorescent compounds derived from 5-cyano-1,6-dihydro-4-methyl-2-phenyl-6thioxopyrimidine," Dyes and Pigments, vol. 82, no. 1, pp. 6-12, 2009.

[30] Y. W. Ho, "Synthesis of new 2-[(Substituted-pyrazolin-1yl)carbonyl]-thieno[2,3-b]pyridine derivatives," Journal of the Chinese Chemical Society, vol. 46, no. 1, pp. 91-96, 1999.

[31] M. Abid and A. Azam, "1-N-substituted thiocarbamoyl-3phenyl-2-pyrazolines: synthesis and in vitro antiamoebic activities," European Journal of Medicinal Chemistry, vol. 40, no. 9, pp. 935-942, 2005. 

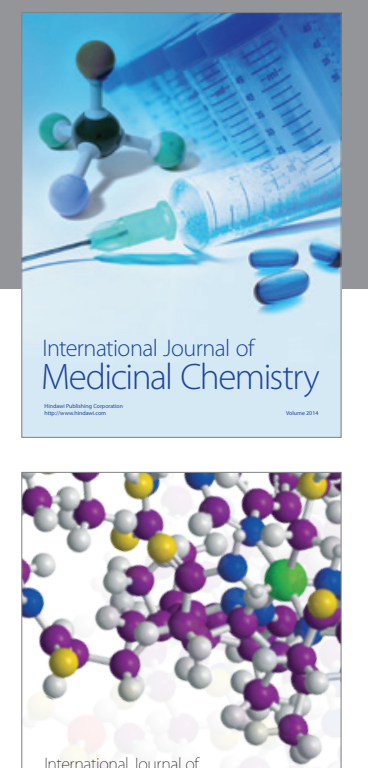

\section{Carbohydrate} Chemistry

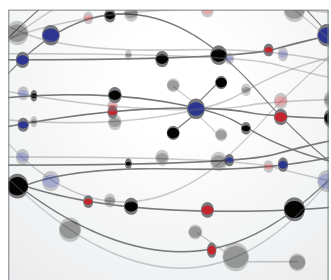

The Scientific World Journal
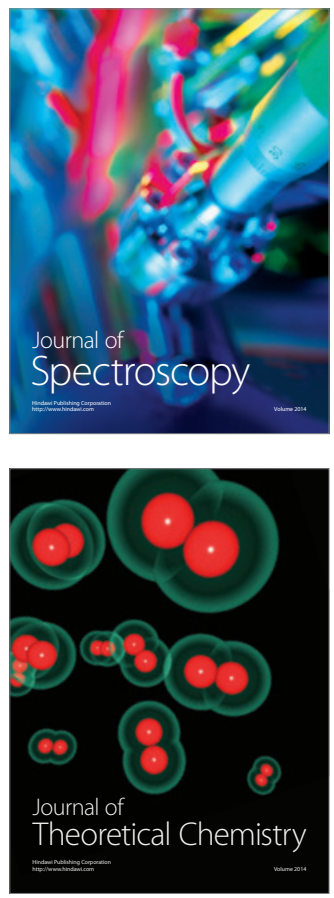
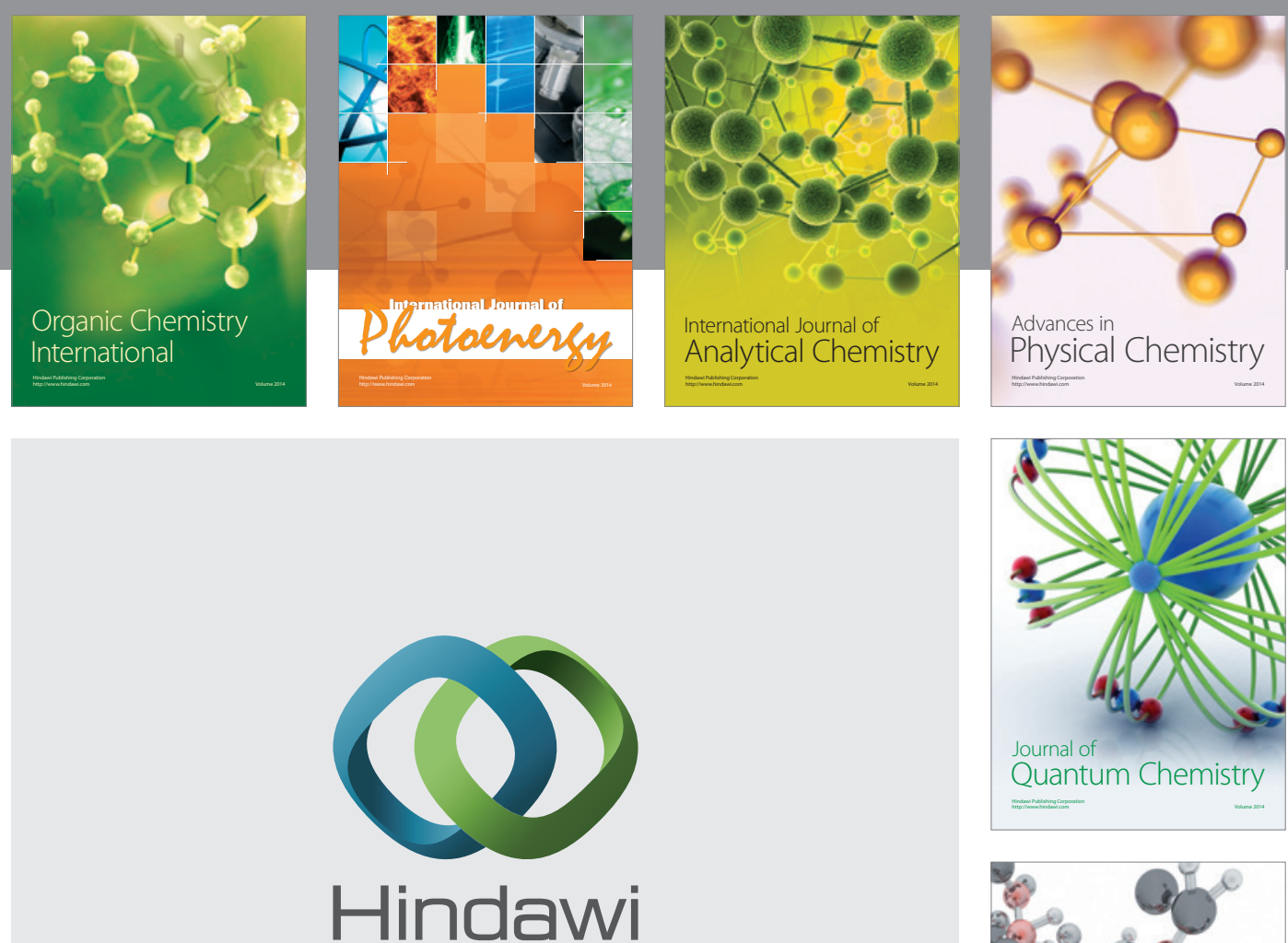

Submit your manuscripts at

http://www.hindawi.com

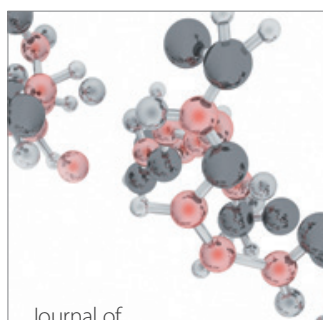

Analytical Methods

in Chemistry

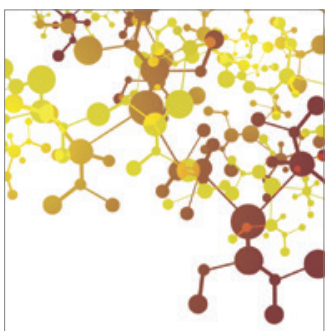

Journal of

Applied Chemistry

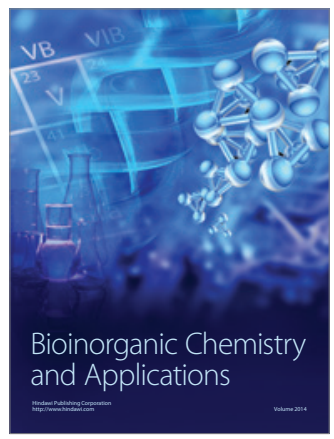

Inorganic Chemistry
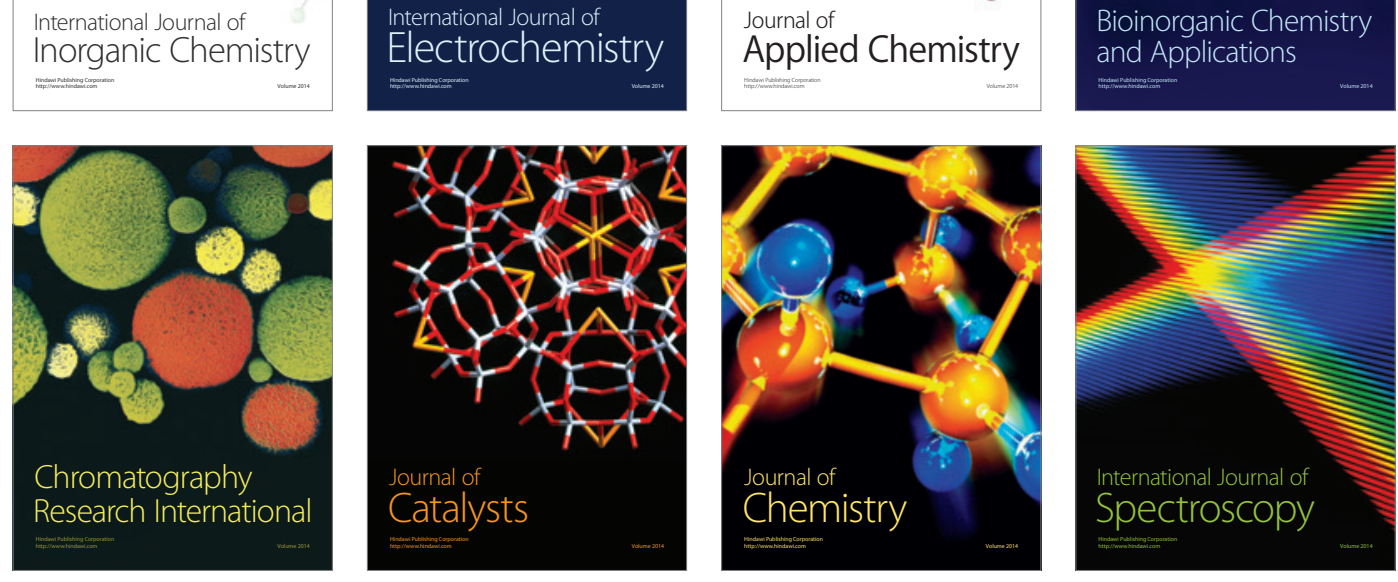